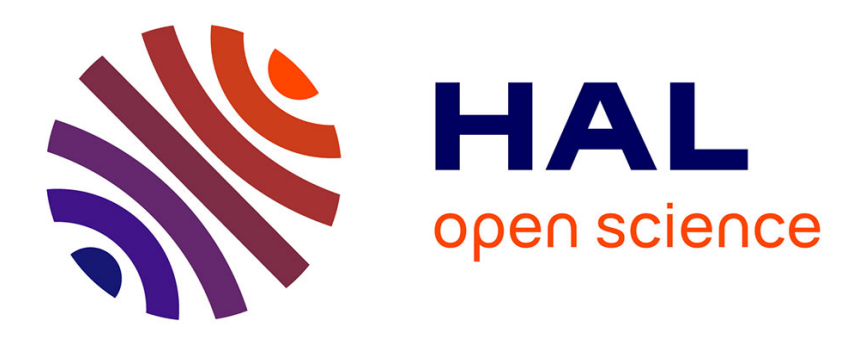

\title{
Characterizations of Preferential Entailments
}

Yves Moinard, Raymond Rolland

\section{To cite this version:}

Yves Moinard, Raymond Rolland. Characterizations of Preferential Entailments. [Research Report] RR-3928, INRIA. 2000. inria-00072724

\section{HAL Id: inria-00072724 \\ https://hal.inria.fr/inria-00072724}

Submitted on 24 May 2006

HAL is a multi-disciplinary open access archive for the deposit and dissemination of scientific research documents, whether they are published or not. The documents may come from teaching and research institutions in France or abroad, or from public or private research centers.
L'archive ouverte pluridisciplinaire HAL, est destinée au dépôt et à la diffusion de documents scientifiques de niveau recherche, publiés ou non, émanant des établissements d'enseignement et de recherche français ou étrangers, des laboratoires publics ou privés. 
INSTITUT NATIONAL DE RECHERCHE EN INFORMATIQUE ET EN AUTOMATIQUE

\section{Characterizations of Preferential Entailments}

Yves Moinard, Raymond Rolland

$\mathbf{N}^{\circ} 3928$

Avril 2000

THÈME 3 



\title{
RINRIA
}

\section{Characterizations of Preferential Entailments}

\author{
Yves Moinard *, Raymond Rolland ${ }^{\dagger}$ \\ Thème 3 - Interaction homme-machine, \\ images, données, connaissances \\ Projet AÏDA \\ Rapport de recherche $\mathrm{n}^{\circ} 3928$ - Avril $2000-26$ pages
}

\begin{abstract}
A preferential entailment is defined by a binary relation, or "preference relation". This relation may be among interpretations, or sets of interpretations, or among "states" which are "copies" of interpretations or of sets of interpretations. This provides four kinds of preferential entailments. The paper deals mainly with propositional logic, however it describes also the situation in first order logic. For instance, the third notion presented above can be seen as a way to simulate the main aspects of the simplest first notion when it is defined in the predicate calculus, while staying in the propositional calculus. Indeed, the third notion allows as many copies of interpretations as we want for a complete theory, even in the propositional calculus, and this is precisely what happens in the predicate calculus. What we do here is to provide a characterization result for these four kinds of preferential entailments. We choose properties as simple and natural as possible, and sometimes we provide various characterizations for the same notion. It appears that the apparently most complicated notion possesses by far the simplest characterization result. A by-product of our results is that the fourth notion is equivalent to the second notion: we may define directly the relation among sets of interpretations, eliminating the need for "states" in this case.
\end{abstract}

Key-words: Preferential entailment, non monotonic reasoning, common sense reasoning, knowledge representation, propositional logic.

(Résumé : tsvp)

This is the research report version of a paper submitted in January, 2000

* IRISA, Campus de Beaulieu, 35042 RENNES-Cedex FRANCE, tel.: (33) 2998473 13, e-mail: moinard@irisa.fr

† IRMAR, Campus de Beaulieu, 35042 RENNES-Cedex FRANCE, tel.: (33) 2992860 19, e-mail: Raymond.Rolland@univ-rennes1.fr

Unité de recherche INRIA Rennes

IRISA, Campus universitaire de Beaulieu, 35042 RENNES Cedex (France)

Téléphone : 0299847100 - International : +33299847100

Télécopie : 0299847171 - International : +33299847171 


\section{Caractérisations des inférences préférentielles}

Résumé : Une inférence préférentielle est définie par une relation binaire, appelée "relation de préférence". Cette relation peut être définie sur des interprétations, ou sur des ensembles d'interprétations, ou sur des "états" qui sont des "copies" d'interprétations ou d'ensembles d'interprétations. Cela donne donc quatre versions d'inférences préférentielles. Ce papier se concentre sur le cas de la logique propositionnelle, mais il décrit également la situation dans le cas de la logique du premier ordre. Ainsi, la troisième version introduite ci-dessus peut être considérée comme une manière de simuler les principaux aspects de la version la plus simple (la première) quand elle est définie dans le cadre du calcul des prédicats, tout en demeurant en logique propositionnelle: en effet, cette troisième version autorise dans le cas propositionnel autant de copies d'interprétations que l'on veut pour une théorie complète, ce qui est la situation du calcul des prédicats. Ce papier fournit des résultats de caractérisation, en termes de propriétés logiques purement syntaxiques, pour les quatre versions d'inférence préférentielle. À chaque fois, les propriétés les plus simples et naturelles possibles sont choisies, et parfois plusieurs résultats de caractérisation sont fournis pour la même notion. Une conséquence de ces résultats est que la quatrième version n'est en réalité pas plus générale que la deuxième: on peut définir directement la relation sur les ensembles d'interprétations, sans avoir recours aux "états" dans ce cas.

Mots-clé : Inférence préférentielle, raisonnement non monotone, raisonnement de sens commun, représentation des connaissances, logique propositionnelle. 


\section{Contents}

1 Introduction 4

2 Notations and framework 4

3 The two simplest preferential entailments 5

4 A small menagerie of properties 6

5 General preferential entailments $\quad 7$

5.1 Definition and first properties ...................... 7

5.2 A characterization of general preferential entailments . . . . . . . . . 9

6 Characterizing multi-preferential entailments $\quad 11$

6.1 Another property of pre-circumscriptions ............... 11

6.2 Para-circumscriptions .................... 12

6.3 The characterization result, in terms of pre-circumscription . . . . . . . . 14

7 Characterizing preferential entailments $\quad 16$

7.1 A property of preferential entailments . . . . . . . . . . . . . . 16

7.2 Characterizing preferential entailments . . . . . . . . . . . 17

8 The first order case $\quad 21$

9 Conclusion $\quad 25$ 


\section{Introduction}

The notion of preferential entailment has shown to be very useful in knowledge representation, when dealing with some aspects of common sense reasoning such as implicit knowledge or rules with exceptions. Preferential entailments are particular inference operations, and various kinds exist. The general idea is as follows: we are given some amount of (certain) knowledge, represented as a set of formulas. This set can be associated with various kinds of objects. For instance, it can be associated with the set of its models, or equivalently in the propositional case, with the set of the complete theories which entail the given formulas. More generally, it can also be associated with the set of all the theories which entail the given formulas. Then we are given a binary relation among the objects associated with our knowledge, and we keep only the objects which are "preferred" for this relation, meaning the minimal ones. Then, we get additional formulas, which are considered as deduced "by default": in the absence of other information, we conclude that we get also all the formulas associated to this reduced set of objects. This allows to reason in a non monotonic way, as augmenting the certain knowledge may invalidate some conclusions previously made by defaults, because some new objects which were not minimal may be minimal in the smaller set associated with the new certain knowledge. We can allow more flexibility by using copies of models, or copies of theories, defining the relation among these sets of "copies". We get then four kinds of preferential entailments. We show below that no real additional flexibility is obtained by replacing the theories by copies of theories, which leaves three kinds. We provide characterization results, in terms of syntactical properties, for these three kinds. The most general version has the simplest characterization result. Characterizations were known for particular cases of these three (or four) versions, but not for the general case.

In section 2 we introduce the notations. We work in propositional logic. In sections 3 and 4 , we give a few reminders about the simplest kinds of preferential entailments. In section 5 we introduce the characterization result for the most general version of preferential entailment. Then, we give in sections 6 and 7 respectively the characterization results for the two "simplest kinds". In section 8, we translate our results to the first order case.

\section{Notations and framework}

- $\mathbf{L}, V, \varphi, \mathcal{T}$ : We work in a propositional language $\mathbf{L}$, assimilated to its set of formulas. $V(\mathbf{L})$, the vocabulary of $\mathbf{L}$, denotes a set of propositional symbols. Letters $\varphi, \psi$ denote formulas in L. A formula will be assimilated to its equivalence class. Letters such as $\mathcal{T}$ or $\mathcal{C}$ denote sets of formulas.

- $\mathbf{M}, \mu, \mathcal{P}(E), \mu \models \cdots$ : Letters $\mu, \nu$ denote interpretations for $\mathbf{L}$. An interpretation is assimilated to the subset of $V(\mathbf{L})$ that it satisfies. The writings $\mu \models \varphi$ and $\mu \models \mathcal{T}$ are defined classically. As usual, for any set $E, \mathcal{P}(E)$ denotes the set of all the subsets of $E$. The set $\mathcal{P}(V(\mathbf{L}))$ of all the interpretations for $\mathbf{L}$ is denoted by $\mathbf{M}$. A model of $\mathcal{T}$ is an interpretation $\mu$ such that $\mu \models \mathcal{T} . \mathbf{M}(\mathcal{T})$ and $\mathbf{M}(\varphi)$ denote the sets of all the models of $\mathcal{T}$ and $\varphi$ respectively. 
- $\mathcal{T} \models \cdots, T h(\mathcal{T}), \mathbf{T}: \mathcal{T} \models \varphi$ and $\mathcal{T} \models \mathcal{T}_{1}$ are defined classically by $\mathbf{M}(\mathcal{T}) \subseteq \mathbf{M}(\varphi)$ and $\mathbf{M}(\mathcal{T}) \subseteq \mathbf{M}\left(\mathcal{T}_{1}\right)$ respectively. A theory is a subset of $\mathbf{L}$ closed for deduction, and for any $\mathcal{T} \subseteq \mathbf{L}$ we denote by $T h(\mathcal{T})$ the set $\{\varphi \in \mathbf{L}, \mathcal{T} \models \varphi\}$. We denote by $\mathbf{T}$ the set $\{\mathcal{T} \subseteq \mathbf{L}, T h(\mathcal{T})=\mathcal{T}\}$ of the theories of $\mathbf{L}$. When $\mathcal{T}_{1}$ is in $\mathbf{T}$, for $\varphi \in \mathbf{L}$ and $\mathcal{T}_{2} \subseteq \mathbf{L}$, we have $\mathcal{T}_{1} \models \varphi$ iff $\varphi \in \mathcal{T}_{1}$ and $\mathcal{T}_{1} \models \mathcal{T}_{2}$ iff $\mathcal{T}_{2} \subseteq \mathcal{T}_{1}$.

- $T, \perp$, ப: Two logical constants $T$ and $\perp$ denote respectively the true and the false formulas. If $V(\mathbf{L})=\emptyset$, then $\mathbf{L}=\{\top, \perp\}$. For any $\mathbf{L}, T h(\emptyset)=T h(\top)=\{\top\}$ and $T h(\perp)=\mathbf{L} \supseteq\{\top, \perp\}$. If $\mathcal{T}_{1}, \mathcal{T}_{2}$ are subsets of $\mathbf{L}$, and $\varphi$ is a formula in $\mathbf{L}$, we write $\mathcal{T}_{1} \sqcup \mathcal{T}_{2}$ for the theory $\operatorname{Th}\left(\mathcal{T}_{1} \cup \mathcal{T}_{2}\right)$ and $\mathcal{T} \sqcup \varphi$ for the theory $\operatorname{Th}(\mathcal{T} \cup\{\varphi\})$. We define similarly $\bigsqcup_{i \in I} \mathcal{T}_{i}=$ $T h\left(\bigcup_{i \in I} \mathcal{T}_{i}\right)$. For any $\mathbf{T}_{1} \subseteq \mathbf{T}$, we have then $\mathbf{M}\left(\bigsqcup_{\mathcal{T} \in \mathbf{T}_{i}} \mathcal{T}\right)=\mathbf{M}\left(\bigcup_{\mathcal{T} \in \mathbf{T}_{i}} \mathcal{T}\right)=\bigcap_{\mathcal{T} \in \mathbf{T}_{i}} \mathbf{M}(\mathcal{T})$. Remember that we have also $\bigcup_{\mathcal{T} \in \mathbf{T}_{i}} \mathbf{M}(\mathcal{T}) \subseteq \mathbf{M}\left(\bigcap_{\mathcal{T} \in \mathbf{T}_{i}} \mathcal{T}\right)$, with equality if $\mathbf{T}_{1}$ is finite.

- $\mathbf{C}, T h(\mu), T h\left(\mathbf{M}_{1}\right)$ : A theory $\mathcal{C} \in \mathbf{T}$ is complete if, for any $\varphi \in \mathbf{L}, \varphi \in \mathcal{C}$ iff $\neg \varphi \notin \mathbf{C}$. We denote by $\mathbf{C}$ the set of all the complete theories of $\mathbf{L}$. Th( $\mu)$ denotes the set of the formulas satisfied by $\mu$. For any subset $\mathbf{M}_{1}$ of $\mathbf{M}, T h\left(\mathbf{M}_{1}\right)=\{\varphi \in \mathbf{L}, \mu \models \varphi$ for any $\mu \in$ $\left.\mathbf{M}_{1}\right\}=\bigcap_{\mu \in \mathbf{M}_{1}} T h(\mu)$. This ambiguous use of $T h$ and of $\models$ (applied to sets of formulas or interpretations) is usual in logic and should not provoke confusion. For any $\mathcal{T} \in \mathbf{T}$, we get $\mathcal{T}=\bigcap_{\mathcal{C} \in \mathbf{C}, \mathcal{C}=\mathcal{T}} \mathcal{C}$. $\mathbf{C}$ may be assimilated to $\mathbf{M}$ : For any $\mu \in \mathbf{M}$ we have $T h(\mu) \in \mathbf{C}$ and for any $\mathcal{C} \in \mathbf{C}, \mathbf{M}(\mathcal{C})$ is a singleton $\{\mu\} \subseteq \mathbf{M}$.

- $T C: T C$ denotes the topological closure: $T C\left(\mathbf{M}_{1}\right)=\mathbf{M}\left(T h\left(\mathbf{M}_{1}\right)\right)$ for $\mathbf{M}_{1} \subseteq \mathbf{M}$. A subset $\mathbf{M}_{1}$ of $\mathbf{M}$ is equal to $\mathbf{M}(\mathcal{T})$ for some $\mathcal{T}$ iff it is closed (i.e. $T C\left(\mathbf{M}_{1}\right)=\mathbf{M}_{1}$ ). A subset $\mathbf{M}_{1}$ of $\mathbf{M}$ is the set of the models of a formula $\varphi$ in $\mathbf{L}$ (i.e., of a finitely axiomatizable theory $\mathcal{T}$ of $\mathbf{L})$ iff it is open and closed, i.e. $T C\left(\mathbf{M}_{1}\right)=\mathbf{M}_{1}$ and $T C\left(\mathbf{M}-\mathbf{M}_{1}\right)=\mathbf{M}-\mathbf{M}_{1}$. If $\mathbf{M}_{1} \subseteq \mathbf{M}$ and $\varphi \in \mathbf{L}$, then $T C\left(\mathbf{M}_{1} \cap \mathbf{M}(\varphi)\right)=T C\left(\mathbf{M}_{1}\right) \cap \mathbf{M}(\varphi)$. If $V(\mathbf{L})$ is finite, any subset of $\mathbf{M}$ is trivially closed and open.

- Neighborhoods: For any $\mu \in \mathbf{M},\{\mathbf{M}(\varphi) / \mu \models \varphi\}$ is a base of (open and closed) neighborhoods of $\mu$. Indeed, let $\mathbf{M}_{o}$ be an open subset of $\mathbf{M}$, then $\mathbf{M}_{o}=\mathbf{M}-\mathbf{M}(\mathcal{T})$ for some $\mathcal{T} \in \mathbf{T}$. We get $\mu \in \mathbf{M}_{o}$ iff $\mu \notin \mathbf{M}(\mathcal{T})$ iff there exists $\varphi \in \mathcal{T}$ such that $\mu \not \models \varphi$ (i.e., $\mu \models \neg \varphi$ ). We have $\varphi \in \mathcal{T}$ iff $\mathbf{M}(\varphi) \supseteq \mathbf{M}(\mathcal{T})$, iff $\mathbf{M}(\neg \varphi) \subseteq \mathbf{M}_{o}$. This proves that for any open set $\mathbf{M}_{o}$ such that $\mu \in \mathbf{M}_{o}$, there exists some formula $\varphi^{\prime}(=\neg \varphi)$ satisfied by $\mu$, such that $\mathbf{M}\left(\varphi^{\prime}\right) \subseteq \mathbf{M}_{o}$. This means that the set $\left\{\mathbf{M}\left(\varphi^{\prime}\right) / \mu \models \varphi^{\prime}\right\}$ is a base of neighborhoods of $\mu$. $\square$

\section{The two simplest preferential entailments}

Any "preferential entailment" is an inference operation that we call a "pre-circumscription":

Definition 3.1 A pre-circumscription $f$ (in $\mathbf{L}$ ) is an extensive (i.e., $f(\mathcal{T}) \supseteq \mathcal{T}$ for any $\mathcal{T}$ ) mapping from $\mathbf{T}$ to $\mathbf{T}$. For any subset $\mathcal{T}$ of $\mathbf{L}$, we use the abbreviation $f(\mathcal{T})=f(T h(\mathcal{T})$ ), assimilating a pre-circumscription to a particular extensive mapping from $\mathcal{P}(\mathbf{L})$ to itself. We write $f(\varphi)$ for $f(\{\varphi\})=f(T h(\varphi))$. 
Definitions 3.2 1 . A preference relation in $\mathbf{L}$ is a binary relation $\prec$ over $\mathbf{M}$. $\mathbf{M}_{\prec}(\mathcal{T})$ denotes the set of the models of $\mathcal{T}$ minimal for $\prec$ :

$\mathbf{M}_{\prec}(\mathcal{T})=\{\mu \in \mathbf{M}(\mathcal{T}) /$ for no $\nu \in \mathbf{M}(\mathcal{T})$ we have $\nu \prec \mu\}$.

2. The (classical) preferential entailment $f=f_{\prec}$ is the pre-circumscription in $\mathbf{L}$ defined by

$$
\varphi \in f_{\prec}(\mathcal{T}) \text { if } \mu \models \varphi \text { for any } \mu \in \mathbf{M}_{\prec}(\mathcal{T}) \text {. }
$$

Definition 3.2 is the definition of preferential entailments originating in [11], but transposed in the propositional case. In the predicate calculus, any complete theory has as many models as we want (we must even go outside the notion of set) which makes this notion more powerful. We can simulate in the propositional case the main aspects of the preferential entailments in the predicate calculus case, as those considered in [11], thanks to a notion introduced in [5, Definition 5.6], called here "multi-preferential entailment":

Definitions 3.3 1. $\mathbf{S}$ is some set of copies of elements of $\mathbf{M}$, called states: there exists a mapping $l$ from $\mathbf{S}$ to $\mathbf{M}$, and for any $\mu \in \mathbf{M}$ we call the set $l^{-1}(\mu)=\left\{\mu_{1}, \mu_{2}, \cdots\right\}$ the set (possibly empty) of the copies of the interpretation $\mu$ in $\mathbf{S}$. If $\mathbf{M}$ is finite, we can assume that $\mathbf{S}$ is finite [5]. $\mathbf{S}(\mathcal{T})$ is the subset of $\mathbf{S}$ defined by $\mathbf{S}(\mathcal{T})=l^{-1}(\mathbf{M}(\mathcal{T}))=$ $\{s \in \mathbf{S} / l(s) \in \mathbf{M}(\mathcal{T})\}$.

2. A multi-preference relation in $\mathbf{L}$ is a binary relation $\prec_{m}$ over such a set $\mathbf{S}$. For any $\mathcal{T} \in \mathbf{T}$, we define the sets $\mathbf{S}_{\prec_{m}}(\mathcal{T})=\left\{\mu_{i} \in \mathbf{S}(\mathcal{T}) /\right.$ for no $\nu_{j} \in \mathbf{S}(\mathcal{T})$, we have $\left.\nu_{j} \prec_{m} \mu_{i}\right\}$ and $\mathbf{M}_{\prec_{m}}(\mathcal{T})=l\left(\mathbf{S}_{\prec_{m}}(\mathcal{T})\right)=\left\{l\left(\mu_{i}\right) / \mu_{i} \in \mathbf{S}_{\prec_{m}}(\mathcal{T})\right\}$.

3. A multi-preferential entailment is a pre-circumscription defined by:

$$
\varphi \in f_{\prec m}(\mathcal{T}) \text { if } \mu \models \varphi \text { for any } \mu \in \mathbf{M}_{\prec_{m}}(\mathcal{T}) .
$$

Remarks 3.4 1. Any preferential entailment is a multi-preferential entailment: choose $\mathbf{S}=\mathbf{M}$ and $l=i d e n t i t y$.

2. $f_{\prec_{m}}(\mathcal{T})=T h\left(\mathbf{M}_{\prec_{m}}(\mathcal{T})\right)=\bigcap_{\mu \in \mathbf{M}_{\prec m}(\mathcal{T})} T h(\mu)$.

3. $\quad \mathbf{M}\left(f_{\prec_{m}}(\mathcal{T})\right)=T C\left(\mathbf{M}_{\prec m}(\mathcal{T})\right)$.

$3^{\prime}$. Thus, when $V(\mathbf{L})$ is finite, we get: $\mathbf{M}\left(f_{\prec_{m}}(\mathcal{T})\right)=\mathbf{M}_{\prec_{m}}(\mathcal{T})$.

\section{A small menagerie of properties}

Definitions 4.1 Here are various properties a pre-circumscription may possess. $\varphi$ is a formula in $\mathbf{L}, \mathcal{T}, \mathcal{T}^{\prime \prime}$ are sets of formulas in $\mathbf{L}$, while $\mathcal{T}_{1}, \mathcal{T}_{2}$ are in $\mathbf{T}$ :

Idempotence:

Reverse monotony:

$$
\begin{gathered}
f(f(\mathcal{T}))=f(\mathcal{T}) . \\
f\left(\mathcal{T} \cup \mathcal{T}^{\prime \prime}\right) \subseteq f(\mathcal{T}) \sqcup \mathcal{T}^{\prime \prime} .
\end{gathered}
$$


Case reasoning:

$$
f\left(\mathcal{T}_{1}\right) \cap f\left(\mathcal{T}_{2}\right) \subseteq f\left(\mathcal{T}_{1} \cap \mathcal{T}_{2}\right)
$$

Disjunctive coherence:

$$
f\left(\mathcal{T}_{1} \cap \mathcal{T}_{2}\right) \subseteq f\left(\mathcal{T}_{1}\right) \sqcup f\left(\mathcal{T}_{2}\right)
$$

Cumulative transitivity:

if $\mathcal{T}^{\prime \prime} \subseteq f(\mathcal{T})$ then $f\left(\mathcal{T} \cup \mathcal{T}^{\prime \prime}\right) \subseteq f(\mathcal{T})$.

Cumulative monotony: if $\mathcal{T}^{\prime \prime} \subseteq f(\mathcal{T})$ then $f(\mathcal{T}) \subseteq f\left(\mathcal{T} \cup \mathcal{T}^{\prime \prime}\right)$.

Cumulativity:

if $\mathcal{T}^{\prime \prime} \subseteq f(\mathcal{T})$ then $f(\mathcal{T})=f\left(\mathcal{T} \cup \mathcal{T}^{\prime \prime}\right)$.

(CUMU)

Definitions 4.2 We also need some weaker versions.

1. Formula versions

(RM1) $\quad f(\mathcal{T} \sqcup \varphi) \subseteq f(\mathcal{T}) \sqcup \varphi$.

(CR1) $f(\mathcal{T} \sqcup \varphi) \cap f(\mathcal{T} \sqcup \psi) \subseteq f(\mathcal{T} \sqcup \varphi \vee \psi)$

2. Formula-only versions

(RM0) $f(\psi \wedge \varphi) \subseteq f(\psi) \sqcup \varphi$.

(CR0) $f(\varphi) \cap f(\psi) \subseteq f(\varphi \vee \psi)$.

Each of these properties has an immediate interpretation in term of reasoning. For instance, (CR1) means that if we know that generally birds fly, formalized as $F l y_{i} \in f(\mathcal{T} \sqcup$ $\left.\operatorname{Bird}_{i}\right)$, and that generally bats fly, formalized as $F l y_{i} \in f\left(\mathcal{T} \sqcup B a t_{i}\right)$, then if all we know a priori about individual $i$ is that it is a bird or a bat, we conclude that $i$ flies $\left(F l y_{i} \in\right.$ $\left.f\left(\mathcal{T} \sqcup \operatorname{Bird}_{i} \vee B a t_{i}\right)\right)$.

Property 4.3 [known] For any pre-circumscription:

1. (RM1) and (CR1) are equivalent, as are (RM0) and (CR0).

2. (RM) implies (CT) and (CR), (CT) implies (Idem).

Here is an important property that a (multi) preference relation may possess.

Definition 4.4 A (multi) preference relation $\prec$ satisfies the closure property (or is (cl)), if for any $\mathcal{T} \in \mathbf{T}, \mathbf{M}_{\prec}(\mathcal{T})$ is a closed set: $\mathbf{M}_{\prec}(\mathcal{T})=\mathbf{M}\left(f_{\prec}(\mathcal{T})\right)$.

(cl) is definability preserving in [10], close to fullness property in [4], and faithful in [6]. (Multi) preferential entailments in which the relation is (cl) are simpler because we get $\mathbf{M}\left(f_{\prec}(\mathcal{T})\right)=\mathbf{M}_{\prec}(\mathcal{T})$. If $V(\mathbf{L})$ is finite, $(\mathrm{cl})$ is trivially satisfied.

\section{General preferential entailments}

\subsection{Definition and first properties}

Definitions 5.1 1 . For any $\mathcal{T} \subseteq \mathbf{L}$ we define the subset of $\mathbf{T}: \mathbf{W}(\mathcal{T})=\left\{\mathcal{T}_{1} \in \mathbf{T} / \mathcal{T} \subseteq\right.$ $\left.\mathcal{T}_{1}\right\}$. We write $\mathbf{W}(\varphi)$ for $\mathbf{W}(\{\varphi\})$. $\mathbf{S}$ is some set of copies of elements of $\mathbf{T}$, also called 
states: there exists a mapping $l$ from $\mathbf{S}$ to $\mathbf{T}$ and for any $\mathcal{T} \in \mathbf{T}$ we call the subset $l^{-1}(\mathcal{T})$ of $\mathbf{S}$, the set (possibly empty) of the copies of the theory $\mathcal{T}$ in $\mathbf{S}$.

As usual, we define $l(\mathbf{S})=\{l(s)\}_{s \in \mathbf{S}}=\left\{\mathcal{T} \in \mathbf{T} / l^{-1}(\mathcal{T}) \neq \emptyset\right\}$. For any $\mathcal{T} \subseteq \mathbf{L}, \mathbf{S}(\mathcal{T})$ is the subset of $\mathbf{S}$ defined by $\mathbf{S}(\mathcal{T})=l^{-1}(\mathbf{W}(\mathcal{T}))=\{s \in \mathbf{S} / l(s) \in \mathbf{W}(\mathcal{T})\}$.

2. A general preference relation $\prec_{g}$ is a binary relation over $\mathbf{S}$. If $\mathcal{T} \in \mathbf{T}$, we define the sets $\mathbf{S}_{\prec_{g}}(\mathcal{T})=\left\{s \in \mathbf{S}(\mathcal{T}) / s_{1} \prec_{g} s\right.$ for no $\left.s_{1} \in \mathbf{S}(\mathcal{T})\right\}$, and $\mathbf{W}_{\prec_{g}}(\mathcal{T})=l\left(\mathbf{S}_{\prec_{g}}(\mathcal{T})\right)$. The general preferential entailment $f_{\prec_{g}}$ is defined by

$$
\varphi \in f_{\prec_{g}}(\mathcal{T}) \text { if } \mathcal{T}_{i} \models \varphi \text { for any } \mathcal{T}_{i} \in \mathbf{W}_{\prec_{g}}(\mathcal{T}) .
$$

This notion appears in [5, Definitions 3.11 and 3.13], [4, Definition 4.26] (with some restriction on $\prec_{g}$ ), and [3, Definitions 3.1 and 3.2] (without restriction). In [5, 4], $l_{k l m}$ is a mapping from $\mathbf{S}$ to $\mathcal{P}(\mathbf{M})$, but, for the propositional case this makes no difference [3]: it suffices to define $l(s)=T h\left(l_{k l m}(s)\right)$. Here are two other formulations, obviously equivalent to definition 5.1-3:

Remark 5.2 For any $\varphi \in \mathbf{L}, \varphi \in f_{\prec_{g}}(\mathcal{T})$ iff $\mathbf{W}_{\prec_{g}}(\mathcal{T}) \subseteq \mathbf{W}(\varphi)$.

$f_{\prec_{g}}$ is a pre-circumscription and we have, for any $\mathcal{T} \subseteq \mathbf{L}: f_{\prec_{g}}(\mathcal{T})=\bigcap_{\mathcal{T}_{i} \in \mathbf{W}_{\prec g}(\mathcal{T})} \mathcal{T}_{i}$.

Here are three remarks about this definition:

Remarks 5.3 1. Any multi-preferential entailment is a general preferential entailment: it suffices to take $l(\mathbf{S}) \subseteq \mathbf{C}$, i.e. to take a mapping $l$ from $\mathbf{S}$ to $\mathbf{C} \subseteq \mathbf{T}$.

2. If $\mathbf{M}$ is finite, a simple counting argument (or theorem 5.10 below) shows that we can assume without loss of generality that $\mathbf{S}$ is finite in definition 5.1.

3. In any case, definition 5.1 could be simplified (see definition 5.7 and theorem 5.10 below) without loss of generality: we could take $\mathbf{S}=\mathbf{T}$ and $l=i d e n t i t y$. However, the connexion with multi-preferential entailments given in point 1 is not so immediate with this simplified form.

Lemma 5.4 For any subsets $\mathcal{T}_{1}, \mathcal{T}_{2}$ of $\mathbf{L}$ we have:

1. $\mathcal{T}_{2} \subseteq f_{\prec_{g}}\left(\mathcal{T}_{1}\right)$ iff $\mathbf{W}_{\prec_{g}}\left(\mathcal{T}_{1}\right) \subseteq \mathbf{W}\left(\mathcal{T}_{2}\right)$.

2. If $\mathbf{W}_{\prec_{g}}\left(\mathcal{T}_{1}\right) \subseteq \mathbf{W}\left(\mathcal{T}_{2}\right)$ then $\mathbf{W}_{\prec_{g}}\left(\mathcal{T}_{1}\right) \subseteq \mathbf{W}_{\prec_{g}}\left(\mathcal{T}_{1} \cup \mathcal{T}_{2}\right)$.

Proof: 1 . If $\mathcal{T}_{2} \subseteq f_{\prec_{g}}\left(\mathcal{T}_{1}\right)$, then for any $\varphi \in \mathcal{T}_{2}$ we have $\mathbf{W}_{\prec_{g}}\left(\mathcal{T}_{1}\right) \subseteq \mathbf{W}(\varphi)$, thus $\mathbf{W}_{\prec g}\left(\mathcal{T}_{1}\right) \subseteq \bigcap_{\varphi \in \mathcal{T}_{2}} \mathbf{W}(\varphi)$. We have clearly $\bigcap_{\varphi \in \mathcal{T}_{2}} \mathbf{W}(\varphi)=\mathbf{W}\left(\mathcal{T}_{2}\right)$. If $\mathbf{W}_{\prec_{g}}\left(\mathcal{T}_{1}\right) \subseteq \mathbf{W}\left(\mathcal{T}_{2}\right)$, then for any $\varphi \in \mathcal{T}_{2}$ we get $\mathbf{W}_{\prec_{g}}\left(\mathcal{T}_{1}\right) \subseteq \mathbf{W}(\varphi)$, i.e., $\varphi \in f_{\prec_{g}}\left(\mathcal{T}_{1}\right)$. (This result extends remark 5.2 to theories which are not finitely axiomatizable.)

2. $\mathbf{W}_{\prec_{g}}\left(\mathcal{T}_{1}\right) \subseteq \mathbf{W}\left(\mathcal{T}_{2}\right)$ and $\mathcal{T} \in \mathbf{W}_{\prec_{g}}\left(\mathcal{T}_{1}\right)$ : Then there exists $s \in \mathbf{S}_{\prec_{g}}\left(\mathcal{T}_{1}\right)$ such that $l(s)=\mathcal{T} \in \mathbf{W}\left(\mathcal{T}_{2}\right) \cap \mathbf{W}\left(\mathcal{T}_{1}\right)=\mathbf{W}\left(\mathcal{T}_{1} \cup \mathcal{T}_{2}\right)$. If $s^{\prime} \in \mathbf{S}\left(\mathcal{T}_{1} \cup \mathcal{T}_{2}\right)=\mathbf{S}\left(\mathcal{T}_{1}\right) \cap \mathbf{S}\left(\mathcal{T}_{2}\right)$ we get $s^{\prime} \nprec_{g} s$ from $s \in \mathbf{S}_{\prec_{g}}\left(\mathcal{T}_{1}\right)$, thus $\mathcal{T} \in \mathbf{W}_{\prec_{g}}\left(\mathcal{T}_{1} \cup \mathcal{T}_{2}\right)$. 
Property 5.5 Any general preferential entailment $f=f_{\prec_{g}}$ is a pre-circumscription satisfying $(\mathrm{CT})$.

Proof: Let us suppose $\mathcal{T}_{2} \subseteq f\left(\mathcal{T}_{1}\right)$, i.e. (lemma 5.4-1), $\mathbf{W}_{\prec_{g}}\left(\mathcal{T}_{1}\right) \subseteq \mathbf{W}\left(\mathcal{T}_{2}\right)$. We suppose also $\varphi \in f\left(\mathcal{T}_{1} \cup \mathcal{T}_{2}\right)$, i.e. from remark 5.2, $\mathbf{W}_{\prec_{g}}\left(\mathcal{T}_{1} \cup \mathcal{T}_{2}\right) \subseteq \mathbf{W}(\varphi)$. Then from lemma 5.4-2 we get $\mathbf{W}_{\prec_{g}}\left(\mathcal{T}_{1}\right) \subseteq \mathbf{W}(\varphi)$, i.e., $\varphi \in f\left(\mathcal{T}_{1}\right)$ : this establishes $f\left(\mathcal{T}_{1} \cup \mathcal{T}_{2}\right) \subseteq f\left(\mathcal{T}_{1}\right)$, thus $f$ satisfies $(\mathrm{CT})$.

Any general preferential entailment satisfies (CT). We will prove the converse and provide a simple form for general preferential entailments, which shows that this notion is "overly defined", and that it looks more "cumbersome" [5] than it is.

\subsection{A characterization of general preferential entailments}

Definition 5.6 Let us call simplified any general preference relation $\prec_{g}$ where $\mathbf{S}=\mathbf{W}$ and $l$ is the identity. In this case, the set $\mathbf{S}$ of states is useless.

Definition 5.7 $f$ being a pre-circumscription in $\mathbf{L}$, we define three relations on the set $\mathbf{T}$ :

$\mathcal{T}_{1} \prec_{1} \mathcal{T}_{2}$ if $f\left(\mathcal{T}_{2}\right)=\mathcal{T}_{1}$ and $\mathcal{T}_{1} \neq \mathcal{T}_{2}$.

$\mathcal{T}_{1} \prec_{2} \mathcal{T}_{2}$ if $\mathcal{T}_{1} \subset \mathcal{T}_{2}$ and $f\left(\mathcal{T}_{1}\right) \notin \mathcal{T}_{2}$.

$\mathcal{T}_{1} \prec_{f} \mathcal{T}_{2}$ if $\mathcal{T}_{1} \prec_{1} \mathcal{T}_{2}$ or $\mathcal{T}_{1} \prec_{2} \mathcal{T}_{2}$.

Here are a few immediate consequences of these definitions:

Remark 5.8 $1 . \prec_{1}, \prec_{2}$ and $\prec_{f}$ are irreflexive.

If $f$ satisfies (Idem), $\prec_{1}$ is trivially transitive: we have never $\mathcal{T}_{1} \prec_{1} \mathcal{T}_{2} \prec_{1} \mathcal{T}_{3}$.

$\prec_{2}$ and $\prec_{f}$ are not necessarily transitive.

2. Let us suppose that we have $\mathcal{T}_{0} \prec_{f} \mathcal{T}$ for some $\mathcal{T}_{0}, \mathcal{T}$ in $\mathbf{T}$ :

- We have $\mathcal{T}_{0} \prec_{1} \mathcal{T}$ iff $\mathcal{T} \subseteq \mathcal{T}_{0}$; in this case we have $\mathcal{T} \subset f(\mathcal{T})=\mathcal{T}_{0}$.

- We have $\mathcal{T}_{0} \prec_{2} \mathcal{T}$ iff $\mathcal{T} \nsubseteq \mathcal{T}_{0}$.

Lemma 5.9 If $\mathcal{T}^{\prime} \in \mathbf{W}_{\prec_{g}}(\mathcal{T}) \subseteq \mathbf{W}\left(\mathcal{T}^{\prime}\right)$ for some $\mathcal{T}^{\prime} \in \mathbf{T}$, then $f_{\prec_{g}}(\mathcal{T})=\mathcal{T}^{\prime}$.

Proof: From $\mathbf{W}_{\prec_{g}}(\mathcal{T}) \subseteq \mathbf{W}\left(\mathcal{T}^{\prime}\right)$ we get $\bigcap_{\mathcal{T}_{1} \in \mathbf{W}_{\prec g}(\mathcal{T})} \mathcal{T}_{1} \supseteq \mathcal{T}^{\prime}$. From $\mathcal{T}^{\prime} \in \mathbf{W}_{\prec_{g}}(\mathcal{T})$ we get then $\mathcal{T}^{\prime}=\bigcap_{\mathcal{T}_{1} \in \mathbf{W}_{\prec g}(\mathcal{T})} \mathcal{T}_{1}=f_{\prec g}(\mathcal{T})$.

Theorem 5.10 If $f$ is a pre-circumscription satisfying $(\mathrm{CT})$, then $f=f_{\prec_{f}}$.

Proof: 1) $f(\mathcal{T}) \in \mathbf{W}_{\prec_{f}}(\mathcal{T}):$ Let us suppose $f(\mathcal{T})=\mathcal{T}$. For no $\mathcal{T}_{0}$ we have $\mathcal{T}_{0} \prec_{1} \mathcal{T}$. As for any $\mathcal{T}_{0} \in \mathbf{W}(\mathcal{T})$ we have $\mathcal{T} \subseteq \mathcal{T}_{0}$, for no $\mathcal{T}_{0} \in \mathbf{W}(\mathcal{T})$ we have $\mathcal{T}_{0} \prec_{2} \mathcal{T}$. Thus, we get $f(\mathcal{T})=\mathcal{T} \in \mathbf{W}_{\prec_{f}}(\mathcal{T})$.

Let us suppose now $f(\mathcal{T}) \neq \mathcal{T}$. Defining $\mathcal{T}_{0}=f(\mathcal{T})$, we get $f\left(\mathcal{T}_{0}\right)=\mathcal{T}_{0}$ from (Idem), a consequence of (CT) (property 4.3-2). Thus, for no $\mathcal{T}^{\prime} \in \mathbf{T}$ we have $\mathcal{T}^{\prime} \prec_{1} \mathcal{T}_{0}$. Let us suppose that there exists $\mathcal{T}^{\prime} \in \mathbf{W}(\mathcal{T})$ such that $\mathcal{T}^{\prime} \prec_{2} \mathcal{T}_{0}$. Then $\mathcal{T} \subseteq \mathcal{T}^{\prime}$ from $\mathcal{T}^{\prime} \in \mathbf{W}(\mathcal{T})$,

$\mathrm{RR} \mathrm{n}^{\circ} 3928$ 
and $\mathcal{T}^{\prime} \subset \mathcal{T}_{0}$ and $f\left(\mathcal{T}^{\prime}\right) \nsubseteq \mathcal{T}_{0}$ from $\mathcal{T}^{\prime} \prec_{2} \mathcal{T}_{0}$. We get $\mathcal{T}^{\prime} \subset f(\mathcal{T})$, and, from (CT), $f\left(\mathcal{T} \sqcup \mathcal{T}^{\prime}\right) \subseteq f(\mathcal{T})$ with $\mathcal{T} \sqcup \mathcal{T}^{\prime}=\mathcal{T}^{\prime}$, thus $f\left(\mathcal{T}^{\prime}\right) \subseteq f(\mathcal{T})$, i.e., $f\left(\mathcal{T}^{\prime}\right) \subseteq \mathcal{T}_{0}$, a contradiction.

2) $\mathbf{W}_{\prec f}(\mathcal{T}) \subseteq \mathbf{W}(f(\mathcal{T}))$ : Let us suppose $\mathcal{T}_{0} \in \mathbf{W}_{\prec_{f}}(\mathcal{T})$. We get $\mathcal{T}_{0} \in \mathbf{W}(\mathcal{T})$, i.e., $\mathcal{T} \subseteq \mathcal{T}_{0}$. If $\mathcal{T}_{0} \neq \mathcal{T}$ we get $f(\mathcal{T}) \subseteq \mathcal{T}_{0}$, as otherwise we would get $\mathcal{T} \prec_{2} \mathcal{T}_{0}$, a contradiction with $\mathcal{T}_{0} \in \mathbf{W}_{\prec_{f}}(\mathcal{T})$. If $\mathcal{T}_{0}=\mathcal{T}$ then, if $\mathcal{T} \neq f(\mathcal{T})$, we get $f(\mathcal{T}) \prec_{1} \mathcal{T}$, a contradiction with $\mathcal{T}=\mathcal{T}_{0} \in \mathbf{W}_{\prec f}(\mathcal{T})$. Thus, we get $\mathcal{T}_{0}=\mathcal{T}=f(\mathcal{T})$. In any case, we get $f(\mathcal{T}) \subseteq \mathcal{T}_{0}$, i.e. $\mathcal{T}_{0} \in \mathbf{W}(f(\mathcal{T}))$.

3) From 1,2 and lemma 5.9 , we get $f(\mathcal{T})=f_{\prec_{f}}(\mathcal{T})$.

Here are two immediate consequences of property 5.5 and theorem 5.10:

Corollary 5.11 For any general preferential entailment $f$, there exists a simplified general preference relation $\prec_{g s}$ such that $f=f_{\prec_{g s}}$.

Thus, the definition of general preferential entailments could have been simplified. This "elimination of the states" in definition 5.1, was partially known, but only if the general preferential entailment satisfies (CUMU) [1, 2], or another strong condition called "rational monotony" [1]. Our method, which applies to any general preferential entailment, is very simple, and it allows to give directly the result of $f$ from the simplified relation considered here (property 5.13 below).

Here is another immediate consequence of property 5.5 and theorem 5.10. This result extends to any general preferential entailment the characterization already known for general preferential entailments satisfying (CUMU) [5, Theorem 3.25] [4, Theorems 4.30 and 4.35].

Theorem 5.12 A pre-circumscription satisfies (CT) iff it is a general preferential entailment.

Thus, the main difference in terms of logical properties between multi-preferential entailments and general preferential entailments is that the former satisfies (CR) and (CT) (see theorem 6.10 below) while the latter satisfies only (CT). It does not exist till now a characterization result in terms of simple logical properties of this kind for (multi) preferential entailments. Thus, the apparently most intricate version of "preferential entailment" possesses in fact, by far, the simplest characterization in terms of logical properties. We will examine below the "simplest kinds" of preferential entailment. Before that, less us give a last result, which is an immediate consequence of definition 5.7:

Property 5.13 Let $f$ be a general preferential entailment, and $\prec_{f}$ be the relation introduced in definition 5.7, then we have, for any $\mathcal{T} \in \mathbf{T}, f(\mathcal{T})=\mathcal{T}_{0}$ iff

$$
\left(\mathcal{T}_{0} \prec_{1} \mathcal{T}\right) \text { or }\left(\mathcal{T}_{0}=\mathcal{T} \text { and, for no } \mathcal{T}^{\prime} \in \mathbf{T} \text {, we have } \mathcal{T}^{\prime} \prec_{1} \mathcal{T}\right) \text {. }
$$

Remember from remark 5.8-2 that it is easy to get $\prec_{1}$ when we know $\prec_{f}: \mathcal{T}_{0} \prec_{1} \mathcal{T}$ iff $\mathcal{T}_{0} \prec_{f} \mathcal{T}$ and $\mathcal{T} \subseteq \mathcal{T}_{0}$. Thus it is immediate to get $f(\mathcal{T})$ when we know $\prec_{f}$. 
Notice that $\prec_{f}$ is such that for any $\mathcal{T} \in \mathbf{T}$, there exists one and only one $\mathcal{T}_{0} \in \mathbf{T}$ satisfying the conditions given here. An interest of this result is that it shows why the notion of general preferential entailment is not an economical way of defining a pre-circumscription. Indeed, a pre-circumscription $f$ may be defined directly in terms of a binary relation over $\mathbf{T}$, by defining e.g. the relation $\prec^{\prime \prime}$ such that $\mathcal{T}_{0} \prec^{\prime \prime} \mathcal{T}$ iff $f(\mathcal{T})=\mathcal{T}_{0}$ (this relation will generally not be a general preference relation associated to $f$, even if $f$ is a general preferential entailment). Thus, defining a binary relation over $\mathbf{T}$ (or $\mathbf{L}$ in the finite case) should suffice to obtain directly the result. It is far from being so with the original definition of general preferential entailments. With our construction, each time $f$ is a general preferential entailment, we have described a general preference relation associated to $f$, and even a simplified relation, which is such that it is immediate to get the value of $f(\mathcal{T})$ directly from $\mathcal{T}$ and from this relation, without making the computations of definition 5.1 or remark 5.2.

Thus, we have exhibited an easy passage, in the two directions, between the notion of precircumscription satisfying $(\mathrm{CT})$ and the notion of general preferential entailment. Starting from a pre-circumscription $f$ satisfying (CT), we use definition 5.7 and we get a (simplified) general preference relation such that $f=f_{\prec_{g}}$. Starting from a general preferential entailment defined by the simplified general preference relation introduced in definition 5.7, property 5.13 shows how the pre-circumscription can be obtained directly from the relation.

Here is the simplest example of a general preferential entailment which is not a multipreferential entailment, i.e., in the finite case, of a pre-circumscription satisfying (CT) and falsifying (CR).

Example 5.1 $V(\mathbf{L})=\{P\}, f(\varphi)=T h(\perp)$ if $\varphi \in\{\perp, P, \neg P\}, f(\top)=T h(\top)$.

$f$ falsifies (CR): $f(P) \cap f(\neg P) \nsubseteq f(P \vee \neg P) . f$ satisfies (CT): it satisfies (Idem) and $f(\varphi)=T h(\perp)$ if $\mathcal{T}_{1} \subset f(\varphi)$. It is easy to check that $f=f_{\prec_{f}}$ where $\perp \prec_{1} P, \perp \prec_{1} \neg P$, $\prec_{f}=\prec_{1}$ and nothing else (definition $5.7, \prec_{2}$ is empty here).

\section{Characterizing multi-preferential entailments}

\subsection{Another property of pre-circumscriptions}

Definitions 6.1 Let $f$ be a pre-circumscription.

1. $f$ satisfies the property of common points, or (CP) if, for any $\varphi \in \mathbf{L}$ and $\mathcal{T} \subseteq \mathbf{L}$ such that $f(\mathcal{T}) \not \models \neg \varphi$, there exists

$$
\mu \in \mathbf{M}(f(\mathcal{T}) \sqcup \varphi) \text { with } \mu \in \bigcap_{\mathcal{T}^{\prime \prime} \in \mathbf{W}(\mathcal{T}), \mu \in \mathcal{T}^{\prime \prime}} \mathbf{M}\left(f\left(\mathcal{T}^{\prime \prime}\right)\right) .
$$

2. $C p_{f}(\mathcal{T})=\left\{\mu \in \mathbf{M}(f(\mathcal{T})) /\{\mu\}=\mathbf{M}\left(\bigsqcup_{\mathcal{T}^{\prime \prime} \in \mathbf{W}(\mathcal{T}), \mu=\mathcal{T}^{\prime \prime}} f\left(\mathcal{T}^{\prime \prime}\right)\right)\right\}$. 
Despite the formulation given here, $(\mathrm{CP})$ is a "syntactical property". An equivalent formulation in a purely syntactical form is given below in definition 8.4-1.

Property 6.2 1. $C p_{f}(\mathcal{T})=\left\{\mu \in \mathbf{M}(f(\mathcal{T})) / \mu \in \bigcap_{\mathcal{T}^{\prime \prime} \in \mathbf{W}(\mathcal{T}), \mu \mid=\mathcal{T}^{\prime \prime}} \mathbf{M}\left(f\left(\mathcal{T}^{\prime \prime}\right)\right)\right\}$.

2. $f$ satisfies $(\mathrm{CP})$ iff $\mathbf{M}(f(\mathcal{T}))=T C\left(C p_{f}(\mathcal{T})\right)$.

Proof: We have (a) $\left(\mathcal{T}^{\prime \prime} \in \mathbf{W}(\mathcal{T})\right.$ and $\left.\mu \models \mathcal{T}^{\prime \prime}\right)$ iff $\mu \in \mathbf{M}\left(\mathcal{T}^{\prime \prime}\right) \subseteq \mathbf{M}(\mathcal{T})$,

(b) $\bigcap_{\mathcal{T}^{\prime \prime} \in \mathbf{W}(\mathcal{T}), \mu=\mathcal{T}^{\prime \prime}} \mathbf{M}\left(f\left(\mathcal{T}^{\prime \prime}\right)\right)=\mathbf{M}\left(\bigsqcup_{\mathcal{T}}{ }^{\prime \prime} \in \mathbf{W}(\mathcal{T}), \mu=\mathcal{T}^{\prime \prime} \quad f\left(\mathcal{T}^{\prime \prime}\right)\right)$, and

(c) $\mathbf{M}(f(\mathcal{T}) \sqcup \varphi)=\mathbf{M}(f(\mathcal{T})) \cap \mathbf{M}(\varphi)$, thus $f(\mathcal{T}) \not \models \neg \varphi$ iff $\mathbf{M}(f(\mathcal{T})) \cap \mathbf{M}(\varphi) \neq \emptyset$.

1. If $\mu \in \mathbf{M}(f(\mathcal{T}))$, as $\mathbf{M}(f(\mathcal{T})) \subseteq \mathbf{M}(\mathcal{T})$, we have $\mu \in \mathbf{M}(T h(\mu)) \subseteq \mathbf{M}(\mathcal{T})$, i.e. $T h(\mu)$ is one of the $\mathcal{T}^{\prime \prime}$ such that $\mu \in \mathbf{M}\left(\mathcal{T}^{\prime \prime}\right) \subseteq \mathbf{M}(\mathcal{T})$, thus $T h(\mu) \subseteq f(T h(\mu)) \subseteq$ $\bigsqcup_{\mathcal{T}^{\prime \prime} \in \mathbf{W}(\mathcal{T}), \mu=\mathcal{T}^{\prime \prime}} \quad f\left(\mathcal{T}^{\prime \prime}\right)$. So, for any $\mu \in \mathbf{M}(f(\mathcal{T}))$, we have $\mu \in C p_{f}(\mathcal{T})$ iff $\bigsqcup_{\mathcal{T}^{\prime \prime} \in \mathbf{W}(\mathcal{T}), \mu=\mathcal{T}^{\prime \prime}} \quad f\left(\mathcal{T}^{\prime \prime}\right)=T h(\mu) \quad$ iff $\quad \bigsqcup_{\mathcal{T}^{\prime \prime} \in \mathbf{W}(\mathcal{T}), \mu=\mathcal{T}^{\prime \prime}} \quad f\left(\mathcal{T}^{\prime \prime}\right) \subseteq T h(\mu)$, iff $\mu \in$ $\mathbf{M}\left(\bigsqcup_{\mathcal{T}^{\prime \prime} \in \mathbf{W}(\mathcal{T}), \mu=\mathcal{T}^{\prime \prime}} \quad f\left(\mathcal{T}^{\prime \prime}\right)\right)=\bigcap_{\mathcal{T}^{\prime \prime} \in \mathbf{W}(\mathcal{T}), \mu=\mathcal{T}^{\prime \prime}} \quad \mathbf{M}\left(f\left(\mathcal{T}^{\prime \prime}\right)\right)$.

2. $f$ satisfies $(\mathrm{CP})$ is equivalent to

if $\mathbf{M}(f(\mathcal{T})) \cap \mathbf{M}(\varphi) \neq \emptyset$, then $\exists \mu \in \mathbf{M}(\varphi), \mu \in C p_{f}(\mathcal{T})$, i.e. to if $\mathbf{M}(f(\mathcal{T})) \cap \mathbf{M}(\varphi) \neq \emptyset$ then $C p_{f}(\mathcal{T}) \cap \mathbf{M}(\varphi) \neq \emptyset$.

As $C p_{f}(\mathcal{T}) \subseteq \mathbf{M}(f(\mathcal{T}))$, we get $T C\left(C p_{f}(\mathcal{T})\right) \subseteq \mathbf{M}(f(\mathcal{T}))$. Thus, $\mathbf{M}(f(\mathcal{T}))$

$=T C\left(C p_{f}(\mathcal{T})\right)$ is equivalent to $\mathbf{M}(f(\mathcal{T})) \subseteq T C\left(C p_{f}(\mathcal{T})\right) .\{\mathbf{M}(\varphi) / \mu \in \mathbf{M}(\varphi)\}$ is a base of neighborhoods of $\mu$ (see section 2), thus $\mu \in T C\left(C p_{f}(\mathcal{T})\right.$ ) iff for any $\varphi$ such that $\mu \in \mathbf{M}(\varphi)$, $\mathbf{M}(\varphi) \cap C p_{f}(\mathcal{T}) \neq \emptyset$.

We introduce now the notion of para-circumscription, which provides another way to apprehend the notions of pre-circumscription and of reverse monotony.

\subsection{Para-circumscriptions}

Definition 6.3 We call para-circumscription any mapping $G$ from $\mathbf{T}$ to $\mathcal{P}(\mathbf{M})$ such that for any $\mathcal{T} \in \mathbf{T}$, we have: $G(\mathcal{T}) \subseteq \mathbf{M}(\mathcal{T})$.

A para-circumscription $G$ satisfies the property of reverse monotony (for paracircumscriptions) if:

if $\mathcal{T}_{1} \subseteq \mathcal{T}_{2}$, then $G\left(\mathcal{T}_{1}\right) \cap \mathbf{M}\left(\mathcal{T}_{2}\right) \subseteq G\left(\mathcal{T}_{2}\right)$.

(RM-para)

If we assimilate $\mathcal{T}$ to $\mathbf{M}(\mathcal{T})$, any pre-circumscription is a para-circumscription and not conversely.

Property 6.4 Let $\prec_{m}$ be a multi-preference relation and $f$ be a pre-circumscription.

1. $\mathbf{M}_{\prec_{m}}$ is a para-circumscriptions satisfying (RM-para).

2. $C p_{f}$ is a para-circumscription satisfying (RM-para).

3. $f$ satisfies $(\mathrm{RM})$ iff the para-circumscription $\mathbf{M} \circ f$ satisfies (RM-para).

INRIA 
Proof: 1 . Let $\mathcal{T}_{1}$ and $\mathcal{T}_{2}$ be in $\mathbf{T}$ such that $\mathcal{T}_{1} \subseteq \mathcal{T}_{2}$. Let $\mu$ be in $\mathbf{M}_{\prec_{m}}\left(\mathcal{T}_{1}\right) \cap \mathbf{M}\left(\mathcal{T}_{2}\right)$. Any $\nu \in \mathbf{M}\left(\mathcal{T}_{2}\right)$ is in $\mathbf{M}\left(\mathcal{T}_{1}\right)$, and as $\mu \in \mathbf{M}_{\prec m}\left(\mathcal{T}_{1}\right)$, we get $\nu_{i} \nprec_{m} \mu_{j}$ for any $\nu_{i}, \mu_{j}$ in $\mathbf{S}$ such that $l\left(\mu_{j}\right)=\mu$ and $l\left(\nu_{j}\right)=\nu$. Thus $\mu \in \mathbf{M}_{\prec_{m}}\left(\mathcal{T}_{2}\right)$.

2. Let us suppose $\mathcal{T}_{1} \subseteq \mathcal{T}_{2}$, i.e., $\mathbf{M}\left(\mathcal{T}_{2}\right) \subseteq \mathbf{M}\left(\mathcal{T}_{1}\right)$. If $\mu \in C p_{f}\left(\mathcal{T}_{1}\right) \cap \mathbf{M}\left(\mathcal{T}_{2}\right)$, then $\mu \in \mathbf{M}\left(f\left(\mathcal{T}_{1}\right)\right)$ from definition 6.1-2 and $\mu \in \mathbf{M}\left(f\left(\mathcal{T}_{2}\right)\right)$ from property 6.2-1. If $\mu \in C p_{f}\left(\mathcal{T}_{1}\right)$ and if $\mathcal{T}^{\prime \prime}$ is such that $\mu \in \mathbf{M}\left(\mathcal{T}^{\prime \prime}\right) \subseteq \mathbf{M}\left(\mathcal{T}_{2}\right)$, then $\mu \in \mathbf{M}\left(\mathcal{T}^{\prime \prime}\right) \subseteq \mathbf{M}\left(\mathcal{T}_{1}\right)$, and $\mu \in \mathbf{M}\left(f\left(\mathcal{T}^{\prime \prime}\right)\right)$ from property 6.2-1. This establishes (see property 6.2-1) that, if $\mu \in \mathbf{M}\left(f\left(\mathcal{T}_{2}\right)\right) \cap C p_{f}\left(\mathcal{T}_{1}\right)$, then $\mu \in C p_{f}\left(\mathcal{T}_{2}\right)$, thus, if $\mu \in C p_{f}\left(\mathcal{T}_{1}\right) \cap \mathbf{M}\left(\mathcal{T}_{2}\right)$, then $\mu \in C p_{f}\left(\mathcal{T}_{2}\right)$. We have proved that if $\mathcal{T}_{1} \subseteq \mathcal{T}_{2}$, then $C p_{f}\left(\mathcal{T}_{1}\right) \cap \mathbf{M}\left(\mathcal{T}_{2}\right) \subseteq C p_{f}\left(\mathcal{T}_{2}\right)$, i.e., $C p_{f}$ satisfies (RM-para).

3. $f$ satisfies (RM) iff, if $\mathcal{T}_{1} \subseteq \mathcal{T}_{2}$, then $\mathbf{M}\left(f\left(\mathcal{T}_{1}\right)\right) \cap \mathbf{M}\left(\mathcal{T}_{2}\right) \subseteq \mathbf{M}\left(f\left(\mathcal{T}_{2}\right)\right)$.

Property 6.5 To any para-circumscription $G$, we can associate a unique precircumscription $f$ defined by: $\mathbf{M} \circ f=T C \circ G$.

If $G$ is a para-circumscription satisfying (RM-para), then the pre-circumscription $f$ defined by $\mathrm{M} \circ f=T C \circ G$ satisfies $(\mathrm{CP})$.

Proof: The first sentence is obvious.

$\mathbf{M}(f(\mathcal{T})) \cap \mathbf{M}(\varphi)=T C(G(\mathcal{T})) \cap \mathbf{M}(\varphi)=T C(G(\mathcal{T}) \cap \mathbf{M}(\varphi)) . T C(G(\mathcal{T}) \cap \mathbf{M}(\varphi))=\emptyset$ iff $G(\mathcal{T}) \cap \mathbf{M}(\varphi)=\emptyset$. Thus, $\mathbf{M}(f(\mathcal{T})) \cap \mathbf{M}(\varphi) \neq \emptyset$ iff $G(\mathcal{T}) \cap \mathbf{M}(\varphi) \neq \emptyset$. Let $\mu$ be in $G(\mathcal{T}) \cap \mathbf{M}(\varphi)$. For any $\mathcal{T}^{\prime \prime}$ such that $\mu \in \mathbf{M}\left(\mathcal{T}^{\prime \prime}\right) \subseteq \mathbf{M}(\mathcal{T})$ we have $\mathcal{T} \subseteq \mathcal{T}^{\prime \prime}$ and $\mu \in G(\mathcal{T}) \cap \mathbf{M}\left(\mathcal{T}^{\prime \prime}\right)$, thus $\mu \in G\left(\mathcal{T}^{\prime \prime}\right)$ because $G$ satisfies (RM-para), and a fortiori $\mu \in$ $T C\left(G\left(\mathcal{T}^{\prime \prime}\right)\right)=\mathbf{M}\left(f\left(\mathcal{T}^{\prime \prime}\right)\right)$. Thus (see property 6.2-1), $\mu \in C p_{f}(\mathcal{T})$ and $C p_{f}(\mathcal{T}) \cap \mathbf{M}(\varphi) \neq \emptyset$. As in the proof of property 6.2-2, we get then that $f$ satisfies (CP).

The converse also holds: If $f$ satisfies $(\mathrm{CP})$, then $\mathbf{M} \circ f=T C \circ C p_{f}$ and we know that the para-circumscription $C p_{f}$ satisfies (RM-para). This shows that (CP) is closely related (or "corresponds") to the natural property (RM-para).

Definitions 6.6 $G$ is a para-circumscription defined over $\mathbf{L}$.

1. We define the sets of "copies of interpretations" $\mathbf{S}_{1}=\mathbf{M}, \mathbf{S}_{2}=\{(\mu, \mathcal{T}) / \mu \in \mathbf{M}, \mathcal{T} \in$ $\mathbf{T}, \mu \in G(\mathcal{T})\}$, and $\mathbf{S}=\mathbf{S}_{1} \cup \mathbf{S}_{2} . l$ is the mapping from $\mathbf{S}$ to $\mathbf{M}$ defined by: $l(\mu)=$ $l(\mu, \mathcal{T})=\mu$. We define the following multi-preference relation $\prec_{G}$ in $\mathbf{S}: s_{1} \prec_{G} s_{2}$ if

$s_{1}=s_{2} \in \mathbf{S}_{1}$, or

$s_{1}=\mu_{1} \in \mathbf{S}_{1}, \quad s_{2}=\left(\mu_{2}, \mathcal{T}_{2}\right) \in \mathbf{S}_{2}$, and $\mu_{1} \notin \mathbf{M}\left(\mathcal{T}_{2}\right)$.

2. If $\prec_{m}$ is a multi-preference relation, we denote $\prec_{\mathbf{M}_{\prec_{m}}}$ by $\prec_{m}{ }^{\prime}$. $\quad\left[\prec_{m}{ }^{\prime}\right]$

3. If $f$ is a pre-circumscription, we denote $\prec_{\mathrm{M} \circ f}$ by $\prec_{f^{\prime}}$.

$\left[\prec f^{\prime}\right]$

Lemma 6.7 1. If a para-circumscription $G$ satisfies (RM-para), then we have $G=\mathbf{M}_{\prec_{G}}$.

2. For any multi-preference relation $\prec_{m}$, we have $\mathbf{M}_{\prec_{m}}=\mathbf{M}_{\prec_{m}{ }^{\prime}}$.

3. If $f$ is a pre-circumscription satisfying (RM), then $\mathbf{M} \circ f=\mathbf{M}_{\prec_{f}}$.

RR $\quad n^{\circ} 3928$ 
Proof: 1 . - If $\mu \in G(\mathcal{T})$, then $(\mu, \mathcal{T})$ is in $\mathbf{S}_{\prec G}(\mathcal{T})$, thus $l(\mu, \mathcal{T})=\mu$ is in $\mathbf{M}_{\prec G}(\mathcal{T})$. Thus we get $G(\mathcal{T}) \subseteq \mathbf{M}_{\prec G}(\mathcal{T})$.

- If $\mu \in \mathbf{M}_{\prec G}(\mathcal{T})$, then $\mu \in \mathbf{M}(\mathcal{T})$ and $\mu$ has a copy in $\mathbf{S}_{2}, s=\left(\mu, \mathcal{T}^{\prime}\right)$, which is in $\mathbf{S}_{\prec G}(\mathcal{T})$, which means that there exists some $\mathcal{T}^{\prime} \in \mathbf{T}$ such that $\mu \in G\left(\mathcal{T}^{\prime}\right)$. Moreover, for any $s_{1}=\nu \in \mathbf{S}_{1}$, if $\nu \notin \mathbf{M}\left(\mathcal{T}^{\prime}\right)$, then $s_{1} \prec_{G} s$, and, as we have $s \in \mathbf{S}_{\prec G}(\mathcal{T}), l\left(s_{1}\right)=\nu$ cannot be a model of $\mathcal{T}$. Thus, any model $\nu$ of $\mathcal{T}$ is a model of $\mathcal{T}^{\prime}$, i.e. $\mathcal{T}^{\prime} \subseteq \mathcal{T}$. As we have $\mu \in G\left(\mathcal{T}^{\prime}\right) \cap \mathbf{M}(\mathcal{T})$, by (RM-para) we get $\mu \in G(\mathcal{T}): \mathbf{M}_{\prec G}(\mathcal{T}) \subseteq G(\mathcal{T})$.

2. $\mathbf{M}_{\prec_{m}}$ is a para-circumscription satisfying (RM-para) (property 6.4-1). We get then the result from point 1 and from the definition of $\prec_{m}$ '.

3. We know from property 6.4-3 that if $f$ satisfies (RM), then $\mathbf{M} \circ f$ satisfies (RM-para). As in 2, we get then the result that the two para-circumscriptions $\mathbf{M} \circ f$ and $\mathbf{M}_{\prec_{f^{\prime}}}$ are equal from 1 and from definition 6.6.

From lemma 6.7-1 and property $6.4-1$, we get the following result:

Theorem 6.8 (also in [10]) A para-circumscription $G$ corresponds to a multi-preferential entailment iff $G$ satisfies (RM-para), i.e.:

A para-circumscription $G$ is such that there exists some multi-preference relation $\prec_{m}$ such that $G=\mathbf{M}_{\prec_{m}}$ iff $G$ satisfies (RM-para).

This provides a very simple characterization of the notion of multi-preferential entailment in terms of para-circumscription. Notice that this result, extracted from [8] where it is given in the predicate calculus case, has also appeared independently as [10, Proposition 2.83], where para-circumscriptions are called "choice functions satisfying condition (0)" and where (RM-para) is "condition (1)" [10, Part 1.3.5].

\subsection{The characterization result, in terms of pre-circumscription}

The study about para-circumscriptions allows to characterize the notion of multi-preferential entailment in terms of a syntactical property. From lemma 6.7-3 we get that any precircumscription satisfying (RM) is a multi-preferential entailment associated to a multi preference relation $\prec_{f}^{\prime}$ which is (cl). As the converse is immediate, we get easily the following already known [10, Theorem 2.81] characterization result:

Theorem 6.9 A pre-circumscription satisfies (RM) iff it is a multi-preferential entailment defined by a multi-preference relation which is $(\mathrm{cl})$.

Any multi-preferential entailment satisfies two important consequences of (RM):

Theorem 6.10 Any multi-preferential entailment satisfies (CT) and (CR), thus (Idem) and (RM1).

Proof: See [6, Observation 3.4.6] for (CR) and property 5.5 for (CT). However, we think it is instructive to provide the following easy proof (in particular, this shows why property 6.4-1 is stronger than lemma 5.4-2.) Notice that if $V(\mathbf{L})$ is infinite, a multi-preferential 
entailment can falsify (RM), and a pre-circumscription satisfying (CT) and (CR) is not necessarily a multi-preferential entailment (example in [8]).

We suppose that $f=f_{\prec_{m}}$ is a multi-preferential entailment.

We suppose $\mathcal{T} \subseteq \mathcal{T}^{\prime} \subseteq f(\mathcal{T})$ for some $\mathcal{T}, \mathcal{T}^{\prime}$ included in $\mathbf{L}$, i.e., $\mathbf{M}(f(\mathcal{T})) \subseteq \mathbf{M}\left(\mathcal{T}^{\prime}\right) \subseteq$ $\mathbf{M}(\mathcal{T})$. We know that we have $\mathbf{M}_{\prec m}(\mathcal{T}) \subseteq \mathbf{M}(f(\mathcal{T}))$ from remark 3.4-3. Thus we have $\mathbf{M}_{\prec_{m}}(\mathcal{T}) \cap \mathbf{M}\left(\mathcal{T}^{\prime}\right)=\mathbf{M}_{\prec_{m}}(\mathcal{T})$. From property 6.4-1 we get $\mathbf{M}_{\prec_{m}}(\mathcal{T}) \cap \mathbf{M}\left(\mathcal{T}^{\prime}\right) \subseteq \mathbf{M}_{\prec_{m}}\left(\mathcal{T}^{\prime}\right)$, i.e. $\quad \mathbf{M}_{{ }_{m}}(\mathcal{T}) \subseteq \mathbf{M}_{\prec_{m}}\left(\mathcal{T}^{\prime}\right)$. Thus $T C\left(\mathbf{M}_{\prec_{m}}(\mathcal{T})\right) \subseteq T C\left(\mathbf{M}_{\prec_{m}}\left(\mathcal{T}^{\prime}\right)\right)$, i.e. $\mathbf{M}(f(\mathcal{T})) \subseteq$ $\mathbf{M}\left(f\left(\mathcal{T}^{\prime}\right)\right)$, i.e. $f\left(\mathcal{T}^{\prime}\right) \subseteq f(\mathcal{T}): f$ satisfies $(\mathrm{CT})$.

$\mathcal{T}_{1} \in \mathbf{T}, \mathcal{T}_{2} \in \mathbf{T}$. From property $6.4-1$ we get $\mathbf{M}_{\prec_{m}}\left(\mathcal{T}_{1} \cap \mathcal{T}_{2}\right) \cap \mathbf{M}\left(\mathcal{T}_{i}\right) \subseteq \mathbf{M}_{\prec_{m}}\left(\mathcal{T}_{i}\right)$ for $i \in\{1,2\}$. Thus $\mathbf{M}_{\prec m}\left(\mathcal{T}_{1} \cap \mathcal{T}_{2}\right) \cap \mathbf{M}\left(\mathcal{T}_{1} \cap \mathcal{T}_{2}\right) \subseteq \mathbf{M}_{\prec m}\left(\mathcal{T}_{1}\right) \cup \mathbf{M}_{\prec m}\left(\mathcal{T}_{2}\right)$, i.e. $\mathbf{M}_{\prec_{m}}\left(\mathcal{T}_{1} \cap \mathcal{T}_{2}\right) \subseteq \mathbf{M}_{\prec_{m}}\left(\mathcal{T}_{1}\right) \cup \mathbf{M}_{\prec_{m}}\left(\mathcal{T}_{2}\right)$. As $T C$ is a closure, we get $T C\left(\mathbf{M}_{\prec_{m}}\left(\mathcal{T}_{1} \cap \mathcal{T}_{2}\right)\right) \subseteq$ $T C\left(\mathbf{M}_{\prec_{m}}\left(\mathcal{T}_{1}\right) \cup \mathbf{M}_{\prec_{m}}\left(\mathcal{T}_{2}\right)\right)=T C\left(\mathbf{M}_{\prec m}\left(\mathcal{T}_{1}\right)\right) \cup T C\left(\mathbf{M}_{\prec m}\left(\mathcal{T}_{2}\right)\right)$. We get $\mathbf{M}\left(f\left(\mathcal{T}_{1} \cap \mathcal{T}_{2}\right)\right) \subseteq$ $\mathbf{M}\left(f\left(\mathcal{T}_{1}\right)\right) \cup \mathbf{M}\left(f\left(\mathcal{T}_{2}\right)\right)$, i.e. $f\left(\mathcal{T}_{1}\right) \cap f\left(\mathcal{T}_{2}\right) \subseteq f\left(\mathcal{T}_{1} \cap \mathcal{T}_{2}\right): f$ satisfies $(\mathrm{CR})$

The following characterization result is the propositional version of a result of [8] (see section 8 below), which has been given already in [7]:

Theorem 6.11 A pre-circumscription satisfies (CP) iff it is a multi-preferential entailment.

Proof: Let $f$ be a pre-circumscription satisfying (CP), then $\mathbf{M} \circ f=T C \circ C p_{f}$ from property 6.2-2. From property 6.4-2, the para-circumscription $C p_{f}$ satisfies (RM-para), thus (lemma

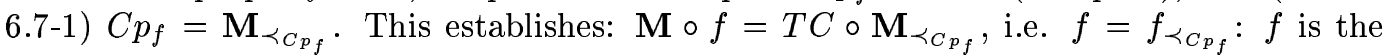
multi-preferential entailment associated to the multi-preference relation $\prec_{C p_{f}}$.

Conversely, let $\prec_{m}$ be a multi-preference relation. We know from property 6.4-1 that $\mathbf{M}_{\prec_{m}}$ is a para-circumscription satisfying (RM-para). With $f=f_{\prec_{m}}$, we have $\mathbf{M} \circ f=$ $T C \circ \mathbf{M}_{\prec_{m}}$ from remark 3.4-3, and $f$ satisfies (CP) from property 6.5.

Remarks 6.12 1. A consequence of theorems 6.10 and 6.11 is that for any precircumscription, $(\mathrm{CP})$ implies $(\mathrm{CR})$ and $(\mathrm{CT})$. (CR) cannot be replaced by $(\mathrm{RM})$ here, and the converse is false: (CR) and (CT) do not imply (CP).

2. A consequence of theorems 6.9 and 6.11 is that for any pre-circumscription, (RM) implies $(\mathrm{CP})$. (RM) cannot be replaced by $(\mathrm{CR})$ or by $\left(\mathrm{P}^{\prime}\right)$ (see definition 7.14 below) $[8]$ and the converse is false: $(\mathrm{CP})$ does not imply (RM).

3. If $V(\mathbf{L})$ is finite, $(\mathrm{CR})$ is equivalent to $(\mathrm{RM})$ (which implies $(\mathrm{CT})$ ) thus, from above results we get that $(\mathrm{CP}),(\mathrm{RM})$ and $(\mathrm{CR})$ are equivalent.

Using theorem 6.11 (or theorem 6.9) we get a simple characterization of multipreferential entailments in the finite case:

If $V(\mathbf{L})$ is finite, a pre-circumscription $f$ is a multi-preferential entailment iff $f$ satisfies $(\mathrm{RM})$ (i.e. $(\mathrm{CR} 0))$. 


\section{Characterizing preferential entailments}

\subsection{A property of preferential entailments}

Definition 7.1 A pre-circumscription $f$ satisfies (DCC) if we have:

$$
f(\mathcal{T}) \subseteq \bigcap_{\mu \in \mathbf{M}(\mathcal{T})} \bigsqcup_{\nu \in \mathbf{M}(\mathcal{T})} f(T h(\{\mu, \nu\}))
$$

(DCC)

If $f$ satisfies the infinite version of (DC) ( $\cap$ and $\sqcup$ ranging over an infinite number of theories), $f$ satisfies (DCC). Thus, if $V(\mathbf{L})$ is finite, (DC) implies (DCC), but in the infinite case, even $(\mathrm{RM})$ and (DC) do not imply (DCC) [8]. Also, examples show that very simple traditional predicate circumscriptions falsify (DCC) [8]. As for (CP), notice that (DCC) is in fact a purely syntactical property: see the equivalent definition given below in definition 8.4-3. (DCC) is fundamental (theorem 7.13 below). Here is another writing for (DCC):

Definition 7.2 Let us denote by $\mathbf{M}_{f}(\mathcal{T})$ the following set of interpretations:

$$
\mathbf{M}_{f}(\mathcal{T})=\{\mu / \mu \in \mathbf{M}(\mathcal{T}), \forall \nu \in \mathbf{M}(\mathcal{T}), \mu \in \mathbf{M}(f(T h(\{\mu, \nu\})))\} .
$$

Property 7.3 1. $\mathbf{M}_{f}(\mathcal{T})=\bigcup_{\mu \in \mathbf{M}(\mathcal{T})} \bigcap_{\nu \in \mathbf{M}(\mathcal{T})} \mathbf{M}(f(T h(\{\mu, \nu\})))$.

2. A pre-circumscription $f$ satisfies (DCC) iff $\mathbf{M}_{f}(\mathcal{T}) \subseteq \mathbf{M}(f(\mathcal{T}))$.

Proof: $1 . \subseteq$ : If $\mu^{\prime \prime} \in \mathbf{M}_{f}(\mathcal{T})$, then $\mu^{\prime \prime} \in \mathbf{M}(\mathcal{T})$ and $\mu^{\prime \prime} \in \bigcap_{\mu^{\prime} \in \mathbf{M}(\mathcal{T})} \mathbf{M}\left(f\left(T h\left(\left\{\mu^{\prime \prime}, \mu^{\prime}\right\}\right)\right)\right) \subseteq \bigcup_{\mu \in \mathbf{M}(\mathcal{T})} \bigcap_{\mu^{\prime} \in \mathbf{M}(\mathcal{T})} \mathbf{M}\left(f\left(T h\left(\left\{\mu, \mu^{\prime}\right\}\right)\right)\right)$.

1. : If $\mu^{\prime \prime} \in \bigcup_{\mu \in \mathbf{M}(\mathcal{T})} \bigcap_{\mu^{\prime} \in \mathbf{M}(\mathcal{T})} \mathbf{M}\left(f\left(T h\left(\left\{\mu, \mu^{\prime}\right\}\right)\right)\right)$, there exists $\mu \in \mathbf{M}(\mathcal{T})$ such that $\mu^{\prime \prime} \in \bigcap_{\mu^{\prime} \in \mathbf{M}(\mathcal{T})} \mathbf{M}\left(f\left(T h\left(\left\{\mu, \mu^{\prime}\right\}\right)\right)\right)$. As $\bigcap_{\mu^{\prime} \in \mathbf{M}(\mathcal{T})} \mathbf{M}\left(f\left(T h\left(\left\{\mu, \mu^{\prime}\right\}\right)\right)\right) \subseteq$ $\mathbf{M}(f(T h(\{\mu, \mu\}))) \subseteq\{\mu\}$, we get $\mu^{\prime \prime} \in\{\mu\}$ thus $\mu^{\prime \prime}=\mu$. Thus $\mu^{\prime \prime}=\mu \in \mathbf{M}_{f}(\mathcal{T})$.

2.: Let us prove another formulation of the result: $f$ satisfies (DCC) iff:

$$
\text { if } \mu \in \mathbf{M}(\mathcal{T}) \text { then } \bigcap_{\mu^{\prime} \in \mathbf{M}(\mathcal{T})} \mathbf{M}\left(f\left(T h\left(\left\{\mu, \mu^{\prime}\right\}\right)\right)\right) \subseteq \mathbf{M}(f(\mathcal{T})) .
$$

Indeed, $f$ satisfies (DCC) iff for any $\mu \in \mathbf{M}(\mathcal{T})$, we get $f(\mathcal{T}) \subseteq$ $\bigsqcup_{\mu^{\prime} \in \mathbf{M}(\mathcal{T})} f\left(T h\left(\left\{\mu, \mu^{\prime}\right\}\right)\right)$, i.e., $\mathbf{M}\left(\bigsqcup_{\mu^{\prime} \in \mathbf{M}(\mathcal{T})} f\left(T h\left(\left\{\mu, \mu^{\prime}\right\}\right)\right)\right) \subseteq \mathbf{M}(f(\mathcal{T}))$.

Now, if we reverse the inclusion in this property equivalent to (DCC), we get another interesting property that a pre-circumscription may possess. This property is given here in three obviously equivalent forms.

Definition 7.4 A pre-circumscription $f$ satisfies the property $(\mathrm{P})$ if:

if $\mu \in \mathbf{M}(f(\mathcal{T}))$ and $\mu^{\prime} \in \mathbf{M}(\mathcal{T})$ then $f\left(T h\left(\left\{\mu, \mu^{\prime}\right\}\right)\right) \subseteq T h(\mu)$, i.e.,

INRIA 


$$
\begin{aligned}
& \text { if } \mu \in \mathbf{M}(f(\mathcal{T})) \text { then } \bigsqcup_{\mu^{\prime} \in \mathbf{M}(\mathcal{T})} f\left(T h\left(\left\{\mu, \mu^{\prime}\right\}\right)\right)=T h(\mu), \quad \text { i.e., } \\
& \mathbf{M}(f(\mathcal{T})) \subseteq \mathbf{M}_{f}(\mathcal{T}) .
\end{aligned}
$$

Property 7.5 For any pre-circumscription, (RM) implies (P).

Proof: If $f(\mathcal{T}) \subseteq T h(\mu)$ and $\mathcal{T} \subseteq T h\left(\left\{\mu, \mu^{\prime}\right\}\right)$ then, by $(\mathrm{RM}), f\left(T h\left(\left\{\mu, \mu^{\prime}\right\}\right)\right) \subseteq$ $f(\mathcal{T}) \bigsqcup T h\left(\left\{\mu, \mu^{\prime}\right\}\right) \subseteq T h(\mu)$. Remember that $T h\left(\left\{\mu, \mu^{\prime}\right\}\right)=T h(\mu) \cap T h\left(\mu^{\prime}\right)$.

Examples show that, even for a multi-preferential entailment, (P) does not imply (RM), however $(\mathrm{P})+(\mathrm{DCC})$ implies $(\mathrm{RM})[8]$. Thus (see theorem 7.13 below), for preferential entailments, $(\mathrm{P})$ is equivalent to (RM). Examples show also that a (multi) preferential entailment may falsify $(\mathrm{P})[8]$.

\subsection{Characterizing preferential entailments}

Definition 7.6 For any pre-circumscription $f$, we define the preference relation $\prec_{f}^{c}$ by:

$$
\begin{aligned}
& \mu \prec_{f}^{c} \nu \quad \text { if } \mu=\nu \text { and } f(T h(\mu)) \neq T h(\mu), \quad \text { or } \quad\left[\prec_{f}^{c}\right] \\
& \mu \neq \nu, f(T h(\nu))=T h(\nu) \text { and } T h(\mu) \subseteq f(T h(\{\mu, \nu\})) .
\end{aligned}
$$

For any multi-preference relation $\prec_{m}$, we define the preference relation $\prec_{m}^{c}=\prec_{f_{\prec_{m}}}^{c} . \quad\left[\prec_{m}^{c}\right]$

Lemma 7.7 For any pre-circumscription $f$ and any $\mathcal{T} \subseteq \mathbf{L}$, we have:

1. $\mu \in \mathbf{M}_{\prec_{f}^{c}}(\mathcal{T})$ iff $\mu \in \mathbf{M}(\mathcal{T})$ and $\mu \in \bigcap_{\mu^{\prime} \in \mathbf{M}(\mathcal{T})} \mathbf{M}_{\prec_{f}^{c}}\left(T h\left(\left\{\mu, \mu^{\prime}\right\}\right)\right.$.

2. For any $\mu, \mu^{\prime}$ in $\mathbf{M}$, we have

$$
\mu \in \mathbf{M}_{\prec_{f}^{c}}\left(T h\left(\left\{\mu, \mu^{\prime}\right\}\right) \text { iff } f(T h(\mu))=T h(\mu) \text { and } f\left(T h\left(\left\{\mu, \mu^{\prime}\right\}\right)\right) \subseteq T h(\mu) .\right.
$$

3. $\mathbf{M}_{f}(\mathcal{T})=\mathbf{M}_{\prec_{f}^{c}}(\mathcal{T})$.

Proof: 1. Immediate. Again, remember that $T h\left(\left\{\mu, \mu^{\prime}\right\}=T h(\mu) \cap T h\left(\mu^{\prime}\right)\right.$.

2. The result is obvious if $\mu=\mu^{\prime}$, thus we can suppose $\mu \neq \mu^{\prime}$. If $\mu$ is in $\mathbf{M}_{\prec_{f}^{c}}\left(T h\left(\left\{\mu, \mu^{\prime}\right\}\right)\right.$, we have $\mu \nprec_{f}^{c} \mu$, thus $f(T h(\mu))=T h(\mu)$. Also, we have $\mu^{\prime} \nprec_{f}^{c}$ $\mu$, thus $T h\left(\mu^{\prime}\right) \nsubseteq f\left(T h\left(\left\{\mu, \mu^{\prime}\right\}\right)\right)$. For any $\mu, \mu^{\prime} \in \mathbf{M}$, we have $f\left(T h\left(\left\{\mu, \mu^{\prime}\right\}\right)\right) \in$ $\left\{\operatorname{Th}\left(\left\{\mu, \mu^{\prime}\right\}\right), T h(\mu), \operatorname{Th}\left(\mu^{\prime}\right), \mathbf{L}\right\}$. Thus, as $\mu \neq \mu^{\prime}$, we have $\operatorname{Th}\left(\mu^{\prime}\right) \nsubseteq f\left(T h\left(\left\{\mu, \mu^{\prime}\right\}\right)\right)$ iff $f\left(T h\left(\left\{\mu, \mu^{\prime}\right\}\right)\right) \subseteq T h(\mu)$.

Conversely, let us suppose $f(T h(\mu))=T h(\mu)$ and $f\left(T h\left(\left\{\mu, \mu^{\prime}\right\}\right)\right) \subseteq T h(\mu)$. We get: $\mu \nprec_{f}^{c} \mu$ and, if $\mu^{\prime} \neq \mu, T h\left(\mu^{\prime}\right) \nsubseteq f\left(T h\left(\left\{\mu, \mu^{\prime}\right\}\right)\right)$, thus $\mu^{\prime} \nprec_{f}^{c} \mu$ and $\mu$ is minimal for $\prec_{f}^{c}$ in $\left\{\mu, \mu^{\prime}\right\}=\mathbf{M}\left(T h\left(\left\{\mu, \mu^{\prime}\right\}\right)\right)$.

3. We prove the following result, which is obviously equivalent to $\mathbf{M}_{f}(\mathcal{T})=\mathbf{M}_{\prec_{f}^{c}}(\mathcal{T})$.

$$
\begin{array}{lll}
\mu \in \mathbf{M}_{\prec_{f}^{c}}(\mathcal{T}) & \text { iff } \quad \mu \in \mathbf{M}(\mathcal{T}) \text { and } \mu \in \bigcap_{\mu^{\prime} \in \mathbf{M}(\mathcal{T})} \mathbf{M}\left(f\left(T h\left(\left\{\mu, \mu^{\prime}\right\}\right)\right)\right), \\
& \text { iff } \quad \mu \in \mathbf{M}(\mathcal{T}) \text { and }\{\mu\}=\bigcap_{\mu^{\prime} \in \mathbf{M}(\mathcal{T})} \mathbf{M}\left(f\left(T h\left(\left\{\mu, \mu^{\prime}\right\}\right)\right)\right) .
\end{array}
$$

Clearly, $\bigcap_{\mu^{\prime} \in \mathbf{M}(\mathcal{T})} \mathbf{M}\left(f\left(T h\left(\left\{\mu, \mu^{\prime}\right\}\right)\right)\right) \subseteq\{\mu\}$. From 1 and 2, if $\mu \in \mathbf{M}(\mathcal{T})$, we get: $\mu \in \mathbf{M}_{\prec{ }_{f}^{c}}(\mathcal{T})$ iff, $f(T h(\mu))=T h(\mu)$ and, for any $\mu^{\prime} \in \mathbf{M}(\mathcal{T}), f\left(T h\left(\left\{\mu, \mu^{\prime}\right\}\right)\right) \subseteq T h(\mu)$, i.e. 
iff, for any $\mu^{\prime} \in \mathbf{M}(\mathcal{T}), f\left(T h\left(\left\{\mu, \mu^{\prime}\right\}\right)\right) \subseteq T h(\mu)$, i.e. iff $\mu \in \bigcap_{\mu^{\prime} \in \mathbf{M}(\mathcal{T})} \mathbf{M}\left(f\left(T h\left(\left\{\mu, \mu^{\prime}\right\}\right)\right)\right)$.

Here are two immediate consequences of lemma 7.7-3.

Property 7.8 A pre-circumscription $f$, satisfies $(\mathrm{P})$ iff $\mathbf{M}(f(\mathcal{T})) \subseteq \mathbf{M}_{\prec_{f}^{c}}(\mathcal{T})$ for any $\mathcal{T} \subseteq$ L.

Property 7.9 If $\prec_{m}$ is a multi-preference relation, with $f=f_{\prec_{m}}$, then $\mathbf{M}_{\prec_{m}^{c}}(\mathcal{T})=\mathbf{M}_{f}(\mathcal{T})$ for any $\mathcal{T} \subseteq \mathbf{L}$.

Here are three important properties that a multi-preference relation may possess.

Definition 7.10 1. A multi-preference relation $\prec_{m}$ is compatible with elementary equivalence, or (cee), if, for any $\mathcal{T} \subseteq \mathbf{L}$ and any elements $s, s_{1}, s^{\prime}, s_{1}^{\prime}$ of $\mathbf{S}$ such that $l(s)=l\left(s^{\prime}\right)$ and $l\left(s_{1}\right)=l\left(s_{1}^{\prime}\right)$, we have $s \prec_{m} s_{1}$ iff $s^{\prime} \prec_{m} s_{1}^{\prime}$.

2. A multi-preference relation $\prec_{m}$ is minimally compatible with elementary equivalence, or (mcee), if, for any $\mathcal{T} \in \mathbf{T}$ and any $s_{1}, s_{2}$ in $\mathbf{S}$ such that $l\left(s_{1}\right)=l\left(s_{2}\right)$, we have $s_{1} \in \mathbf{S}_{\prec m}(\mathcal{T})$ iff $s_{2} \in \mathbf{S}_{\prec m}(\mathcal{T})$.

3. A multi-preference relation $\prec_{m}$ is regular or (reg) if, for any $\mathcal{T} \subseteq \mathbf{L}$, we have: $\mathbf{M}_{f}(\mathcal{T})=$ $\mathbf{M}_{\prec m}(\mathcal{T})$, where $f=f_{\prec m}$.

(cee) implies (mcee) (obvious) and (mcee) implies (reg) (see lemma 7.12 below). The converses are false (examples in [8]). However we will see in theorem 7.13 below that some important weak converses are true.

These properties are all related to the fact that the multi-preferential entailment $f_{\prec_{m}}$ is in fact a preferential entailment (see theorem 7.13 below, this is immediate for (cee)).

Property 7.11 For any multi-preferential entailment $f=f_{\prec_{m}}$ we have, for any $\mathcal{T} \subseteq \mathbf{L}$ :

$$
\mathbf{M}_{\prec m}(\mathcal{T}) \subseteq \mathbf{M}_{f}(\mathcal{T})=\bigcup_{\mu \in \mathbf{M}(\mathcal{T})} \bigcap_{\mu^{\prime} \in \mathbf{M}(\mathcal{T})} \mathbf{M}_{\prec m}\left(T h\left(\left\{\mu, \mu^{\prime}\right\}\right)\right) .
$$

$\underline{\text { Proof: }}=:$ As any subset of $\left\{\mu, \mu^{\prime}\right\}$ is a closed set (being finite), and as $\mathbf{M}_{\prec_{m}}\left(T h\left(\left\{\mu, \mu^{\prime}\right\}\right)\right) \subseteq$ $\left\{\mu, \mu^{\prime}\right\}$, we get $\mathbf{M}_{\prec_{m}}\left(T h\left(\left\{\mu, \mu^{\prime}\right\}\right)\right)=\mathbf{M}\left(f\left(T h\left(\left\{\mu, \mu^{\prime}\right\}\right)\right)\right)$. Then use property 7.3-1.

$\mathbf{M}_{\prec_{m}}(\mathcal{T}) \subseteq \mathbf{M}_{f}(\mathcal{T})$ : If $\mu \in \mathbf{M}_{\prec_{m}}(\mathcal{T})$, then for any $\mu^{\prime} \in \mathbf{M}(\mathcal{T})$, we have $\left.\mathcal{T} \subseteq T h\left(\left\{\mu, \mu^{\prime}\right\}\right)\right)$ and clearly $\mu \in \mathbf{M}\left(T h\left(\left\{\mu, \mu^{\prime}\right\}\right)\right)$. From property $6.4-1$ we get $\mu \in \mathbf{M}_{\prec_{m}}\left(T h\left(\left\{\mu, \mu^{\prime}\right\}\right)\right)$, thus $\mu \in \bigcap_{\mu^{\prime} \in \mathbf{M}(\mathcal{T})} \mathbf{M}_{\prec_{m}}\left(T h\left(\left\{\mu, \mu^{\prime}\right\}\right)\right)$, and, as we have also $\mu \in \mathbf{M}(\mathcal{T}), \mu \in \mathbf{M}_{f}(\mathcal{T})$.

Lemma 7.12 If a multi-preference relation $\prec_{m}$ is (mcee), then it is (reg). 
Proof: If $\mu \in \mathbf{M}_{f}(\mathcal{T})$, then $\mu \in \bigcap_{\mu^{\prime} \in \mathbf{M}(\mathcal{T})} \mathbf{M}_{\prec_{m}}\left(T h\left(\left\{\mu, \mu^{\prime}\right\}\right)\right)$ from definition 7.2 and from the equality $\mathbf{M}_{\prec_{m}}\left(T h\left(\left\{\mu, \mu^{\prime}\right\}\right)\right)=\mathbf{M}\left(f\left(T h\left(\left\{\mu, \mu^{\prime}\right\}\right)\right)\right)$. If $\mu_{i}$ and $\nu_{j}$ are copies of models of $\mathcal{T}$, with $l\left(\mu_{i}\right)=\mu$ and $l\left(\nu_{j}\right)=\nu$, we have $\mathcal{T} \subseteq T h(\{\mu, \nu\})$. If $\nu_{j} \prec_{m} \mu_{i}$, then $\mu_{i} \notin$ $\mathbf{S}_{\prec_{m}}(T h(\{\mu, \nu\}))$ and, from (mcee), no copy $\mu_{k}$ of $\mu$ can be in $\mathbf{S}_{\prec_{m}}(T h(\{\mu, \nu\}))$, thus $\mu \notin$ $\mathbf{M}_{\prec_{m}}(T h(\{\mu, \nu\}))$, which contradicts $\mu \in \bigcap_{\mu^{\prime} \in \mathbf{M}(\mathcal{T})} \mathbf{M}_{\prec_{m}}\left(T h\left(\left\{\mu, \mu^{\prime}\right\}\right)\right)$. Thus, no such $\nu_{j}$ can exist: we get $\mu_{i} \in \mathbf{S}_{\prec_{m}}(\mathcal{T})$, then $\mu \in \mathbf{M}_{\prec_{m}}(\mathcal{T})$. Thus, $\mathbf{M}_{f}(\mathcal{T}) \subseteq \mathbf{M}_{\prec_{m}}(\mathcal{T})$, and, from property 7.11, $\mathbf{M}_{f}(\mathcal{T})=\mathbf{M}_{\prec_{m}}(\mathcal{T})$.

Theorem 7.13 For any multi-preferential entailment $f=f_{\prec_{m}}$, the following properties are equivalent:

(o) $f$ is a preferential entailment.

(i) $f=f_{\prec_{m}^{1}}$ where $\prec_{m}^{1}$ is (cee).

(ii) $f=f_{\prec_{m}^{2}}$ where $\prec_{m}^{2}$ is (mcee).

(iii) $f=f_{\prec_{m}^{3}}$ where $\prec_{m}^{3}$ is (reg).

(iv) $f$ satisfies (DCC).

(v) $f=f_{\prec m^{c}}$.

Proof: $(\mathrm{o}) \Rightarrow$ (i): Any preference relation is trivially (cee).

(i) $\Rightarrow$ (ii): Obvious.

(ii) $\Rightarrow$ (iii): Lemma 7.12.

(iii) $\Rightarrow$ (iv): Remark 3.4-3 and property 7.3-2.

(iv) $\Rightarrow$ (v): We get $\mathbf{M}_{\prec m^{c}}=\mathbf{M}_{f}$ by property 7.9. From (DCC) and property 7.3-2, we get $\mathbf{M}_{\prec_{m^{c}}}(\mathcal{T}) \subseteq \mathbf{M}(f(\mathcal{T}))$, thus $T C\left(\mathbf{M}_{\prec_{m}}(\mathcal{T})\right) \subseteq \mathbf{M}(f(\mathcal{T}))$, i.e. $\mathbf{M}_{f_{\prec_{m}}}(\mathcal{T}) \subseteq \mathbf{M}(f(\mathcal{T}))$. From property 7.11 we get $\mathbf{M}_{\prec_{m}}(\mathcal{T}) \subseteq \mathbf{M}_{f}(\mathcal{T})$, thus $T C\left(\mathbf{M}_{\prec_{m}}(\mathcal{T})\right) \subseteq T C\left(\mathbf{M}_{f}(\mathcal{T})\right)$, i.e. $\mathbf{M}(f(\mathcal{T})) \subseteq \mathbf{M}\left(f_{\prec_{m}}(\mathcal{T})\right)$. We get $\mathbf{M}(f(\mathcal{T}))=\mathbf{M}\left(f_{\prec_{m}}(\mathcal{T})\right)$, i.e. $f=f_{\prec_{m}}$.

(v) $\Rightarrow(\mathrm{o}): \prec_{m}{ }^{c}$ is a preference relation (see definition 7.6).

Here is a direct proof of (i) $\Rightarrow(\mathrm{o})$, which is not necessary for the proof of the theorem, but which is illustrating:

To any multi-preference relation $\prec_{m}$ which is (cee), let us define the following preference relation $\prec: \mu \prec \mu$ if $\mu \notin l(\mathbf{S})$ and $\mu \prec \nu$ if there exists $s_{1}, s_{2}$ in $\mathbf{S}$ such that $l\left(s_{1}\right)=\mu$, $l\left(s_{2}\right)=\nu$ and $s_{1} \prec_{m} s_{2}$ (and nothing else). Then, we get clearly: $f_{\prec_{m}}=f_{\prec}$

Thanks to this theorem, we already get a characterization of preferential entailments, by using also theorem 6.11 . We will improve this result, replacing $(\mathrm{CP})$ by a weaker and simpler condition. Thus, let us introduce, in two equivalent forms, another property that a pre-circumscription may possess.

Definition 7.14 [see definition 7.2] A pre-circumscription $f$ satisfies $\left(\mathrm{P}^{\prime}\right)$ if, for any $\varphi \in \mathbf{L}$ and any $\mathcal{T} \subseteq \mathbf{L}$ we have:

$\mathrm{RR} \mathrm{n}^{\circ} 3928$ 


$$
\begin{aligned}
& \text { if } f(\mathcal{T}) \not \models \neg \varphi \text {, then } \mathbf{M}(f(\mathcal{T}) \sqcup \varphi) \cap \mathbf{M}_{f}(\mathcal{T}) \neq \emptyset \text {; } \\
& \text { i.e.: if } \mathbf{M}(f(\mathcal{T})) \cap \mathbf{M}(\varphi) \neq \emptyset \text { then } \mathbf{M}(f(\mathcal{T})) \cap \mathbf{M}(\varphi) \cap \mathbf{M}_{f}(\mathcal{T}) \neq \emptyset \text {. }
\end{aligned}
$$

Notice that Makinson has independently studied a closely related notion in lemma 3.4.9 in [6], making an usage of this property which is different from us.

\section{Property 7.15 1. Any pre-circumscription satisfying $(\mathrm{P})$ satisfies $\left(\mathrm{P}^{\prime}\right)$.}

2. If $V(\mathbf{L})$ is finite, $(\mathrm{P})$ is equivalent to $\left(\mathrm{P}^{\prime}\right)$.

Proof: 1. If $f$ satisfies $(\mathrm{P})$, we know from definition 7.4 that $\mathbf{M}(f(\mathcal{T})) \subseteq \mathbf{M}_{f}(\mathcal{T})$, thus $\mathbf{M}(f(\mathcal{T})) \cap \mathbf{M}(\varphi)=\mathbf{M}(f(\mathcal{T})) \cap \mathbf{M}(\varphi) \cap \mathbf{M}_{f}(\mathcal{T}): f$ satisfies $\left(\mathrm{P}^{\prime}\right)$.

Even for preferential entailment, $\left(\mathrm{P}^{\prime}\right)$ does not imply $(\mathrm{P})$, i.e., see property 7.16 below, there exist preferential entailments which falsify $(\mathrm{P})[8]$.

2. $V(\mathbf{L})$ is finite, $f$ satisfies $\left(\mathrm{P}^{\prime}\right)$, and $\mu \in \mathbf{M}(f(\mathcal{T}))$. There exists some formula $\varphi \in \mathbf{L}$ such that $\mathbf{M}(\varphi)=\{\mu\}$, i.e. $T h(\mu)=T h(\varphi)$. Thus we have $\mathbf{M}(f(\mathcal{T})) \cap \mathbf{M}(\varphi)=\{\mu\} \neq \emptyset$. From $\left(\mathrm{P}^{\prime}\right)$, we get $\mathbf{M}(f(\mathcal{T})) \cap \mathbf{M}(\varphi) \cap \mathbf{M}_{f}(\mathcal{T}) \neq \emptyset$, thus $\mu \in \mathbf{M}_{f}(\mathcal{T})$ : $f$ satisfies $(\mathrm{P})$.

Property 7.16 Any multi-preferential entailment satisfies $\left(\mathrm{P}^{\prime}\right)$.

Proof: Let us suppose $f=f_{\prec_{m}}$. As $T C\left(\mathbf{M}_{\prec_{m}}(\mathcal{T})\right) \cap \mathbf{M}(\varphi)=T C\left(\mathbf{M}_{\prec_{m}}(\mathcal{T}) \cap \mathbf{M}(\varphi)\right)$ (remember section 2), we get $\mathbf{M}(f(\mathcal{T})) \cap \mathbf{M}(\varphi)=T C\left(\mathbf{M}_{\prec_{m}}(\mathcal{T})\right) \cap \mathbf{M}(\varphi)=T C\left(\mathbf{M}_{\prec_{m}}(\mathcal{T}) \cap \mathbf{M}(\varphi)\right)$. Also, $\mathbf{M}(f(\mathcal{T})) \cap \mathbf{M}(\varphi)=\emptyset$ iff $\mathbf{M}_{\prec_{m}}(\mathcal{T}) \cap \mathbf{M}(\varphi)=\emptyset$. If we suppose $\mathbf{M}(f(\mathcal{T}) \sqcup \varphi)=$ $\mathbf{M}(f(\mathcal{T})) \cap \mathbf{M}(\varphi) \neq \emptyset$, we must have $\mathbf{M}_{\prec_{m}}(\mathcal{T}) \cap \mathbf{M}(\varphi) \neq \emptyset$. From property 7.11 we get $\mathbf{M}_{\prec_{m}}(\mathcal{T}) \subseteq \mathbf{M}_{f}(\mathcal{T})$. From Remark 3.4-3 we get $\mathbf{M}_{\prec_{m}}(\mathcal{T}) \subseteq \mathbf{M}(f(\mathcal{T}))$. Thus, we get a fortiori $\mathbf{M}(f(\mathcal{T})) \cap \mathbf{M}(\varphi) \cap \mathbf{M}_{f}(\mathcal{T}) \neq \emptyset$.

From theorem 6.11, we get then that $(\mathrm{CP})$ implies $\left(\mathrm{P}^{\prime}\right)$. Examples $[8]$ show that there exist pre-circumscriptions satisfying $\left(\mathrm{P}^{\prime}\right)$ which are not multi-preferential entailments.

We can now characterize the notion of preferential entailment (this result, also extracted from [8], has appeared as [7, Proposition 3.8-2]):

Theorem 7.17 A pre-circumscription $f$ is a preferential entailment iff $f$ satisfies (DCC) and $\left(\mathrm{P}^{\prime}\right)$.

Proof: "only if": Any preferential entailment satisfies (DCC) from theorem 7.13 and any (multi-) preferential entailment satisfies $\left(\mathrm{P}^{\prime}\right)$ from property 7.16 .

"if": $f$ satisfies $(\mathrm{DCC})$ and $\left(\mathrm{P}^{\prime}\right)$. By $(\mathrm{DCC})$ we get $\mathbf{M}_{\prec_{f}^{c}}(\mathcal{T}) \subseteq \mathbf{M}(f(\mathcal{T}))$ from properties 7.3-2 and 7.9. Let us suppose now $\mu \in \mathbf{M}(f(\mathcal{T}))$. Then, for any formula $\varphi$ such that $\mu \in \mathbf{M}(\varphi)$, we get $\mathbf{M}(f(\mathcal{T})) \cap \mathbf{M}(\varphi) \cap \mathbf{M}_{f}(\mathcal{T}) \neq \emptyset$ by $\left(\mathrm{P}^{\prime}\right)$. Then, from lemma $7.7-3$, we get $\mathbf{M}(f(\mathcal{T})) \cap \mathbf{M}(\varphi) \cap \mathbf{M}_{\prec_{f}^{c}}(\mathcal{T}) \neq \emptyset$. Thus, for any $\varphi \in \mu$, we have a fortiori $\mathbf{M}(\varphi) \cap \mathbf{M}_{\prec_{f}^{c}}(\mathcal{T}) \neq \emptyset$ and (remember the "neighborhoods" paragraph in section 2) we get $\mu \in T C\left(\mathbf{M}_{\prec_{f}^{c}}(\mathcal{T})\right)$. Thus we have $\mathbf{M}(f(\mathcal{T}))=T C\left(\mathbf{M}_{\prec_{f}^{c}}(\mathcal{T})\right)$, i.e. $f=f_{\prec_{f}^{c}}: f$ is the preferential entailment associated to the preference relation $\prec_{f}^{c}$. We have even established that a pre-circumscription satisfying

INRIA 
(DCC) satisfies $\left(\mathrm{P}^{\prime}\right)$ iff it is a preferential entailment.

Notice that, in the presence of $(\mathrm{DCC})$, the writing of $\left(\mathrm{P}^{\prime}\right)$ can be simplified by dropping the last " $M(f(\mathcal{T})) \cap$ " in the second formulation of $\left(\mathrm{P}^{\prime}\right)$ given in definition 7.14 , thanks to property $7.3-2$.

Examples show that we cannot replace $\left(\mathrm{P}^{\prime}\right)$ by $(\mathrm{P})$ here $[8]$.

Thus, we have shown that in the presence of (DCC), (CP) can be replaced by a simpler property $\left(\mathrm{P}^{\prime}\right)$ (and even a simpler kind of $\left(\mathrm{P}^{\prime}\right)$ ). To our knowledge, $\left(\mathrm{P}^{\prime}\right)$ cannot be replaced by a logical property having an immediate meaning in terms of reasoning, such as (CM), $(\mathrm{CT})$ or (DC). If $V(\mathbf{L})$ is finite, (DC) implies (DCC) while (RM), (CP) and (CR) are equivalent, thus $f$ is a preferential entailment iff it satisfies (DC) and (RM). It can be shown that (RM) and (DCC) imply (DC) (direct proof easy) while in the infinite case (RM) and (DC) do not imply (DCC) [8]. Even if we consider only pre-circumscriptions satisfying (RM), it does not exist, to our knowledge, a simple (meaning eliminating properties such as $\left(\mathrm{P}^{\prime}\right)$ or $(\mathrm{DCC})$ and keeping only properties such as (RM) or (DC)) characterization result for preferential entailments (associated in this case to a relation satisfying (cl)). For what concerns multi-preferential entailments in this case, there is theorem 6.9. It is only in the finite case that there exist simple characterization results for the three kinds of preferential entailment, given just above for the "classical" version, in remark $6.12-3$ for the" multi" version, and by theorem 5.12 for the "general" version.

\section{The first order case}

This work applies also to the first order case: the results about classical and multipreferential entailments come from [8] (first version in August, 1994), which concerns the first order case $^{1} . \mathbf{L}$ is here a first order language and $\mathbf{M}$ denotes the class of all the interpretations for $\mathbf{L}$. $\mathbf{T}$ and $\mathbf{C}$ are as in section 2 . We define a predicate preferential entailment as in definition 3.2. In the propositional case there is a one-to-one mapping between $\mathbf{M}$ and $\mathbf{C}$, thus a propositional preferential entailment could alternatively be defined by a "preference relation" in $\mathbf{C}$ instead of $\mathbf{M}$, and this is what determines the nature of this notion. On the contrary, in the predicate calculus case, any complete theory has many models (we must even go outside the notion of sets, but this is not a concern here). Thus, defining a "preferential entailment" by a relation over the class $\mathbf{M}$ of the interpretations of $\mathbf{L}$, or over the set $\mathbf{C}$ of the complete theories in $\mathbf{L}$, give two different notions.

Definitions 8.1 1. [11] A predicate preference relation $\prec$ is a binary relation over $\mathbf{M}$. The (classical) predicate preferential entailment $f_{\prec}$ is the pre-circumscription defined by

$$
\varphi \in f_{\prec}(\mathcal{T}) \text { if } \mu \models \varphi \text { for any } \mu \in \mathbf{M}_{\prec}(\mathcal{T}) .
$$

\footnotetext{
${ }^{1}$ The predicate versions of these two results, that is of theorems 6.11 and 7.17 , extracted from [8], have been given in [7, Remark 3.9]. As the publication of [8] is still deleted, and as we have now a characterization for all kinds of preferential entailments, including the "general" ones, we have written this short report which contains all the relevant proofs in their propositional version.
} 
Thus we have, as in remark 3.4-2: $f_{\prec}(\mathcal{T})=T h\left(\mathbf{M}_{\prec}(\mathcal{T})\right)=\bigcap_{\mu \in \mathbf{M}_{\prec}(\mathcal{T})} T h(\mu)$.

2. A predicate $\mathbf{C}$-preference relation $\prec_{\mathbf{C}}$ is a binary relation over $\mathbf{C}$. Let us define $\mathbf{C}(\mathcal{T})=$ $\{\mathcal{C} \in \mathbf{C} / \mathcal{T} \subseteq \mathcal{C}\}$. Thus we get, for any $\mathcal{T} \in \mathbf{T}: \mathcal{T}=\bigcap_{\mathcal{C} \in \mathbf{C}(\mathcal{T})} \mathcal{C}$. Let us define $\mathbf{C}_{\prec \mathbf{C}}$ as we have defined $\mathbf{M}_{\prec}(\mathcal{T})$ in definition 3.2: $\mathbf{C}_{\prec \mathbf{C}}(\mathcal{T})=\left\{\mathcal{C} \in \mathbf{C}(\mathcal{T}) /\right.$ for no $\mathcal{C}^{\prime} \in$ $\mathbf{C}(\mathcal{T})$ we have $\left.\mathcal{C}^{\prime} \prec_{\mathbf{C}} \mathcal{C}\right\}$.

The predicate $\mathbf{C}$-preferential entailment $f_{\prec \mathbf{C}}$ is the pre-circumscription defined by

$$
\varphi \in f_{\prec \mathbf{C}}(\mathcal{T}) \text { if } \mathcal{C} \models \varphi \text { for any } \mathcal{C} \in \mathbf{C}_{\prec \mathbf{C}}(\mathcal{T})
$$

Thus we have, as in remark 3.4-2: $f_{\prec \mathbf{C}}(\mathcal{T})=\bigcap_{\mathcal{C} \in \mathbf{C}_{\prec_{\mathbf{C}}}(\mathcal{T})} \mathcal{C}$.

3. A predicate multi-preference relation $\prec_{m}$ is a binary relation over a set $\mathbf{S}$ where there exists a mapping $l$ from $\mathbf{S}$ to $\mathbf{C}$. Let us define $\mathbf{C}_{\prec_{m}}(\mathcal{T}) \subseteq \mathbf{C}$ as we have defined $\mathbf{M}_{\prec_{m}}(\mathcal{T})$ in definition 3.3: $\mathbf{S}(\mathcal{T})=l^{-1}(\mathbf{C}(\mathcal{T}))=\{s \in \mathbf{S} / l(s) \models \mathcal{T}\}, \mathbf{S}_{\prec_{m}}(\mathcal{T})=\{s \in$ $\mathbf{S}(\mathcal{T}) /$ for no $s^{\prime} \in \mathbf{S}(\mathcal{T})$ we have $\left.s^{\prime} \prec_{m} s\right\}$ and $\mathbf{C}_{\prec_{m}}(\mathcal{T})=l\left(\mathbf{S}_{\prec_{m}}(\mathcal{T})\right)=\{l(s) / s \in$ $\left.\mathbf{S}_{\prec m}(\mathcal{T})\right\}$.

The predicate multi-preferential entailment $f_{\prec_{m}}$ is the pre-circumscription defined by

$$
\varphi \in f_{\prec_{m}}(\mathcal{T}) \text { if } \mathcal{C} \models \varphi \text { for any } \mathcal{C} \in \mathbf{C}_{\prec_{m}}(\mathcal{T})
$$

Thus we have, as in remark 3.4-2: $f_{\prec m}(\mathcal{T})=\bigcap_{\mathcal{C} \in \mathbf{C}_{\prec_{m}}(\mathcal{T})} \mathcal{C}$.

Point 1 is the classical definition of "preferential entailments" in the predicate calculus case. For the sake of exhaustivity, a "fourth" kind of preferential entailment should be given, where the relation $\prec_{m}$ is over $\mathbf{S}$, the mapping $l$ being from $\mathbf{S}$ to $\mathbf{M}$ : in this "point 4 ", we would define $\mathbf{M}_{\prec_{m}}$ and $f_{\prec_{m}}$ exactly as in definition 3.3. By the way, we should have named the multi-preferential entailments of point 3 "C-multi-preferential entailments", reserving the name "multi-preferential entailment" for this "fourth point". However, no real power is gain by this definition: this notion would be equivalent to the notion defined in point 3 . It is easy to show this directly, by a method similar to the one used in the proof of lemma 8.2 given below. Another easy proof uses theorem 8.3 given below, and shows that the same characterization result holds for this "fourth notion". Notice however that we have already defined too many kinds of "predicate preferential entailments". Indeed, we will prove now that the notions in points 1 and 3 are equivalent. However, we think it is important to make this matter precise, this is why we have explicitly given the two definitions.

With classical predicate preferential entailments, as well as with multi-preferential entailments, either in the predicate calculus or in the propositional case, we get that any complete theory can be represented by as many "copies" as we want. This justifies the following result:

Lemma 8.2 $f$ is a predicate preferential entailment iff it is a predicate multi-preferential entailment. 
Proof: Let $\prec$ be a predicate preference relation in $\mathbf{M}$ and $f=f_{\prec}$ (definition 8.1-1). Then we have $f_{\prec}(\mathcal{T})=T h\left(\mathbf{M}_{\prec}(\mathcal{T})\right)$. We define $\mathbf{S}=\mathbf{M}$ (it is not a set, but this does not modify the results), and the mapping $l$ from $\mathbf{S}=\mathbf{M}$ to $\mathbf{C}$ by: $l(\mu)=T h(\mu)$. We define $\prec_{m}$ in $\mathbf{S}=\mathbf{M}$ by $\prec_{m}=\prec$. Then we get $\mathbf{C}_{\prec_{m}}(\mathcal{T})=\left\{T h(\mu) / \mu \in \mathbf{M}_{\prec}(\mathcal{T})\right\}$. Thus, $f_{\prec}(\mathcal{T})=f_{\prec_{m}}(\mathcal{T})$.

For the converse, let us suppose that $\prec_{m}$ is a predicate multi-preference relation defined in a set (or a class such as $\mathbf{M}$ ) $\mathbf{S}$. Then, we define a predicate preference relation $\prec$ in $\mathbf{M}$ as follows. Firstly, for any $\mathcal{C} \in l(\mathbf{S}) \subseteq \mathbf{C}$, for each $s \in l^{-1}(\mathcal{C})$ we choose a distinct $\mu \in \mathbf{M}(\mathcal{C})$, denoted by $l^{\prime}(s)$. For any $\mu \in l^{\prime}(\mathbf{S})$, there exists a unique $s \in \mathbf{S}$ with $s=l^{\prime-1}(\mu)$, and $T h(\mu)=T h\left(l^{\prime}(s)\right)=l(s)$. Then, we define $\prec$ in $\mathbf{M}$ by: $\mu \prec \mu$ if $\mu \in \mathbf{M}-l^{\prime}(\mathbf{S})$ and $\mu \prec \nu$ if there exist $s_{1}, s_{2}$ in $\mathbf{S}$ such that $l^{\prime}\left(s_{1}\right)=\mu, l^{\prime}\left(s_{2}\right)=\nu$ and $s_{1} \prec_{m} s_{2}$. We get $\left\{T h(\mu) / \mu \in \mathbf{M}_{\prec}(\mathcal{T})\right\}=\left\{l(s) / s \in \mathbf{S}_{\prec_{m}}(\mathcal{T})\right\}=\mathbf{C}_{\prec_{m}}(\mathcal{T})$. Thus, $f_{\prec}(\mathcal{T})=f_{\prec_{m}}(\mathcal{T})$.

Notice that with the definitions given in this section, a preference relation is not immediately seen as a multi preference relation, however the proof given above shows the close relationship existing between these two kinds of relation. The important difference with the propositional case is that here the two kinds of relation have "the same expressive power".

We get the following characterization of predicate preferential entailments:

Theorem 8.3 [8] A predicate pre-circumscription $f$ is a predicate preferential entailment iff it satisfies $(\mathrm{CP})$.

Sketch of the proof: An automatic translation of the proof given for theorem 6.11 shows that the result applies to predicate multi-preferential entailments (in definition 6.6, we must naturally replace $\mathbf{M}$ by $\mathbf{C})$. Then use lemma $8.2 \square$

As already written, $(\mathrm{CP})$ can be written in terms of theories instead of models. In the predicate calculus case, this writing in terms of theories seems more appropriate. The same holds for (DCC) and $\left(\mathrm{P}^{\prime}\right)$ (if we replace $\mathbf{M}_{f}$ by $\mathbf{C}_{f}$ ) involved in theorem 8.7 below. Here are the precise "syntactical" definitions:

Definition 8.4 $f$ is a pre-circumscription in $\mathbf{L}, \prec$ is a preference relation in $\mathbf{M}$ and $\prec_{m}$ is a multi-preference relation. $\mathcal{T}$ is any subset of $\mathbf{L}$ and $\varphi$ any formula in $\mathbf{L}$.

1. If $f(\mathcal{T}) \not \models \neg \varphi$, there exists $\mathcal{C} \in \mathbf{C}(f(\mathcal{T})) \cap \mathbf{C}(\varphi) \cap \bigcap_{\mathcal{T}_{i} \in \mathbf{T} / \mathcal{C} \in \mathbf{C}\left(\mathcal{T}_{i}\right) \subseteq \mathbf{C}(\mathcal{T})} \mathbf{C}\left(f\left(\mathcal{T}_{i}\right)\right)$. (CP)

2. $C p_{f}(\mathcal{T})=\left\{\mathcal{C} \in \mathbf{C}(f(\mathcal{T})) / \mathcal{C}=\quad \bigsqcup \quad f\left(\mathcal{T}_{i}\right)\right\} . \quad\left[\boldsymbol{C p}_{\boldsymbol{f}}(\mathcal{T})\right]$ $\mathcal{T}_{i} \in \mathbf{T}, \mathcal{C} \in \mathbf{C}\left(\mathcal{T}_{i}\right) \subseteq \mathbf{C}(\mathcal{T})$

(DCC)

3. $f(\mathcal{T}) \subseteq \bigcap_{\mathcal{C} \in \mathbf{C}(\mathcal{T})} \bigsqcup_{\mathcal{C}^{\prime} \in \mathbf{C}(\mathcal{T})} f\left(\mathcal{C} \cap \mathcal{C}^{\prime}\right)$

4. $\mathbf{C}_{f}(\mathcal{T})=\left\{\mathcal{C} \in \mathbf{C}(\mathcal{T}) / \forall \mathcal{C}^{\prime} \in \mathbf{C}(\mathcal{T}), \mathcal{C} \in \mathbf{C}\left(f\left(\mathcal{C} \cap \mathcal{C}^{\prime}\right)\right)\right\}$

5. $\mathbf{C}(f(\mathcal{T})) \subseteq \mathbf{C}_{f}(\mathcal{T})$. 
6. if $f(\mathcal{T}) \not \models \neg \varphi$ then $\mathbf{C}(f(\mathcal{T})) \cap \mathbf{C}(\varphi) \cap \mathbf{C}_{f}(\mathcal{T}) \neq \emptyset$.

$\left(\mathbf{P}^{\prime}\right)$

These definitions are equivalent to (or, for the sets, are in obvious correspondence with) the definitions given in the preceding sections, but in the predicate calculus case we tend to prefer the definitions given here. It is important to notice that, with the definitions given in this section, a predicate preference relation is not necessarily (cee), or (mcee) or (reg). Here are the precise definitions involved (cf definition 7.10):

Definition 8.5 $\prec$ is a preference relation in $\mathbf{M}$ and $\prec_{m}$ is a multi-preference relation.

1. If $T h(\mu)=T h\left(\mu^{\prime}\right)$ and $T h(\nu)=T h\left(\nu^{\prime}\right)$, then $\mu \prec \nu$ iff $\mu^{\prime} \prec \nu^{\prime}$. $\prec$ is (cee)

$1^{\prime}$ If $l(s)=l\left(s^{\prime}\right)$ and $l\left(s_{1}\right)=l\left(s_{1}^{\prime}\right)$, then $s \prec_{m} s_{1}$ iff $s^{\prime} \prec_{m} s_{1}^{\prime}$.

$\prec_{m}$ is (cee)

2. If $T h(\mu)=T h(\nu)$, then for any $\mathcal{T} \subseteq \mathbf{L}, \mu \in \mathbf{M}_{\prec}(\mathcal{T})$ iff $\nu \in \mathbf{M}_{\prec}(\mathcal{T}) . \quad \prec$ is (mcee)

$2^{\prime}$ If $l(s)=l\left(s_{1}\right)$, then for any $\mathcal{T} \subseteq \mathbf{L}, s_{1} \in \mathbf{S}_{\prec_{m}}(\mathcal{T})$ iff $s_{2} \in \mathbf{S}_{\prec_{m}}(\mathcal{T}) . \quad \prec_{m}$ is (mcee)

3. $\mathbf{C}_{f_{\prec}}(\mathcal{T})=\mathbf{C}_{\prec}(\mathcal{T})$ for any $\mathcal{T} \subseteq \mathbf{L}$.

$\prec$ is (reg)

$3^{\prime} \mathbf{C}_{f_{\prec m}}(\mathcal{T})=\mathbf{C}_{\prec m}(\mathcal{T})$ for any $\mathcal{T} \subseteq \mathbf{L}$.

$\prec_{m}$ is (reg)

In definition 7.6, we get in fact a $\mathbf{C}$-preference relation:

Definition 8.6 For any pre-circumscription $f$, we define the $\mathbf{C}$-preference relation $\prec_{f}^{c}$ by:

$$
\begin{aligned}
& C_{1} \prec_{f}^{c} \mathcal{C}_{2} \quad \text { if } \mathcal{C}_{1}=\mathcal{C}_{2} \text { and } f\left(C_{1}\right) \neq \mathcal{C}_{1}, \quad \text { or } \quad\left[\prec_{f}^{c}\right] \\
& \mathcal{C}_{1} \neq \mathcal{C}_{2}, f\left(\mathcal{C}_{2}\right)=\mathcal{C}_{2} \text { and } \mathcal{C}_{1} \subseteq f\left(\mathcal{C}_{1} \cap \mathcal{C}_{2}\right) .
\end{aligned}
$$

For any multi-preference relation $\prec_{m}$, we define the $\mathbf{C}$-preference relation $\prec_{m}^{c}=\prec_{f_{\prec_{m}}^{c}}^{c} \cdot\left[\prec_{m}^{c}\right]$

The translation of theorems 7.17 and 7.13 are as follows (again, the proofs are automatic translations of the proofs given for theorems 7.13 and 7.17):

\section{Theorem $8.7[8]$}

1. A pre-circumscription $f$ in $\mathbf{L}$ is a $\mathbf{C}$-preferential entailment iff it satisfies (DCC) and $\left(\mathrm{P}^{\prime}\right)$.

2. Theorem 7.13 holds, if we replace point (o) by " $f$ is a C-preferential entailment".

Thanks to lemma 8.2, we get even two forms of this theorem, one with (predicate) multi-preferential entailments $f=f_{\prec_{m}}$ as in theorem 7.13, and one when we suppress "multi-", with (predicate) preferential entailments $f=f_{\prec}$.

Well known examples of predicate preferential entailments are predicate circumscriptions. Generally they cannot be defined by a preference relation satisfying (cee), i.e., they falsify (DCC) [8]. This means that they are not C-preferential entailments. A notable (though rather limited) exception is the first order predicate circumscription of one unary predicate, everything else being fixed (see [9] for these definitions), which satisfies (DCC), thus which 
can be defined by a $\mathbf{C}$-preference relation, i.e. by a preference relation which is (cee) [8].

We turn briefly to predicate general preferential entailments now. Theorems 5.10 and 5.12 apply to predicate general preferential entailments defined as in definition 5.1: the states are useless here also. If we define predicate general preference relations as relations among copies of classes of interpretations as done in [5] (though for the propositional case) then we can obtain an equivalent general preference relation defined in $\mathbf{T}$. As in the propositional case, but this is possibly more important in this case (one reason is that a preference relation is defined in the class of all the interpretations of $\mathbf{L}$, while a general preference relation is defined in the "more friendly" set T), any predicate preferential entailment can be defined by a predicate general preference relation.

\section{Conclusion}

We have characterized the three kinds of "preferential entailments" known in the literature, in terms of purely syntactical properties. We have also shown that a fourth version appearing in the literature is in fact overly general, as it can be reduced to one of these three versions. These results show that the most complicate version, where the relation is defined among theories (or sets of interpretations) possesses by far the easiest characterization result. Our results are detailed in the propositional case, but we have seen how to apply them to the first order case as well. Doing so, we have made precise the very close relationship between what we call "multi preferential entailments" (where the relation is among copies of models or of complete theories) in the propositional case and classical preferential entailments (where the relation is directly among models) in the predicate calculus case: these two notions are characterized by exactly the same syntactical property, called "common points". Thus, we have given five results: the characterization of the three kinds of preferential entailment (some of these characterizations appearing in various forms), the identity between a "fourth kind" and a simpler kind of preferential entailment, and the utility of the notion of multi preferential entailment in order to study the classical notion of preferential entailment in the predicate calculus case. To our knowledge, these five results are new: the three characterization results, and the reduction of the fourth kind of preferential entailment to a simpler kind, were known before, but for particular cases only.

A lot of work still remains. A by-product of our results is that any predicate preferential entailment (such as any classical circumscription) can be described as a general preferential entailment, that is by a relation among theories. It remains to investigate this point and to give direct translations from the original relation between interpretations to a relation between theories. Various relations should be studied, in order to find an interesting one which could possibly help our understanding of the notion studied, or the automatic computation. Also, we could try to find some more precise results: when we have a general preferential entailment, are there "easy and natural" conditions, but not too strong, which make that it is in fact a simpler kind of preferential entailment? This amounts to assume cumulative transitivity and to search for a condition, milder than reverse monotony but more friendly than

$\mathrm{RR} \mathrm{n}^{\circ} 3928$ 
$(\mathrm{CP})$ or $\left(\mathrm{P}^{\prime}\right)$, which ensures that we get a simpler preferential entailment. Notice however that our work shows that it is unlikely that we can get really simple and natural properties of the kind of reverse monotony. We should try to find other kinds of properties, still to be discovered (?). Again, the impact of such a study could be on the knowledge representation side, helping to get a better understanding of complex (though useful and natural) notions, and on the computational side.

\section{References}

[1] H. Arlo-Costa and S. Shapiro. Maps between nonmonotonic and conditional logics. In KR'92, pages 553-564, Cambridge, 1992. Morgan Kaufmann.

[2] A. Bochman. An Epistemic Representation of Nonmonotonic Inference. Unpublished manuscript, 1999.

[3] A. Bochman. Credulous Nonmonotonic Inference. In T. Dean, editor, IJCAI-99, pages 30-35, Stockholm, Aug. 1999. Morgan Kaufmann.

[4] M. Freund and D. Lehmann. Nonmonotonic inference operations. Bulletin of the IGPL (Interest Group in Propositional and Predicate Logics), 1(1):23-68, 1993.

[5] S. Kraus, D. Lehmann, and M. Magidor. Nonmonotonic Reasoning, Preferential Models and Cumulative Logics. Artificial Intelligence, 44(1-2):167-207, July 1990.

[6] D. Makinson. General patterns in nonmonotonic reasoning. In D. M. Gabbay, C. Hogger, and J. Robinson, editors, Handbook of Logic in Artificial Intelligence and Logic Programming, Volume 3: Non-Monotonic and Uncertainty Reasoning, pages 35-110. Oxford University Press, 1994.

[7] Y. Moinard and R. Rolland. Propositional circumscriptions. Research Report, INRIA, RR-3538 or IRISA, PI-1211, Rennes, France, Oct. 1998. http://www.inria.fr/RRRT/RR-3538.html.

[8] Y. Moinard and R. Rolland. The logical properties of classical circumscriptions and preferential entailments. Technical report, INRIA, Rennes, France, 2000 (to appear).

[9] D. Perlis and J. Minker. Completeness results for circumscription. Artificial Intelligence, 28(1):29-42, Feb. 1986.

[10] K. Schlechta. Nonmonotonic Logics: Basic Concepts, Results and Techniques, LNAI 1187. Springer-Verlag, Bonn, 1997.

[11] Y. Shoham. Reasoning about change. MIT Press, Cambridge, 1988. 
Unité de recherche INRIA Lorraine, Technopôle de Nancy-Brabois, Campus scientifique, 615 rue du Jardin Botanique, BP 101, 54600 VILLERS LÈS NANCY

Unité de recherche INRIA Rennes, Irisa, Campus universitaire de Beaulieu, 35042 RENNES Cedex Unité de recherche INRIA Rhône-Alpes, 655, avenue de l'Europe, 38330 MONTBONNOT ST MARTIN Unité de recherche INRIA Rocquencourt, Domaine de Voluceau, Rocquencourt, BP 105, 78153 LE CHESNAY Cedex Unité de recherche INRIA Sophia-Antipolis, 2004 route des Lucioles, BP 93, 06902 SOPHIA-ANTIPOLIS Cedex

Éditeur

INRIA, Domaine de Voluceau, Rocquencourt, BP 105, 78153 LE CHESNAY Cedex (France) http://www.inria.fr ISSN 0249-6399 